\title{
The Conserved IgSF9 Protein Borderless Regulates Axonal Transport of Presynaptic Components and Color Vision in Drosophila
}

\author{
난 Hunter S. Shaw, ${ }^{1,2}$ Scott A. Cameron, ${ }^{2,3}$ Wen-Tzu Chang, ${ }^{2}$ and Yong Rao ${ }^{2,3,4}$ \\ ${ }^{1}$ Department of Biology, ${ }^{2} \mathrm{McGill}$ Centre for Research in Neuroscience, ${ }^{3}$ Department of Neurology and Neurosurgery, and ${ }^{4}$ Integrated Program in \\ Neuroscience, McGill University Health Centre, Montreal, Quebec H3G 1A4, Canada
}

\begin{abstract}
Normal brain function requires proper targeting of synaptic-vesicle (SV) and active-zone components for presynaptic assembly and function. Whether and how synaptogenic signals (e.g., adhesion) at axo-dendritic contact sites promote axonal transport of presynaptic components for synapse formation, however, remain unclear. In this study, we show that Borderless (Bdl), a member of the conserved IgSF9-family trans-synaptic cell adhesion molecules, plays a novel and specific role in regulating axonal transport of SV components. Loss of $b d l$ disrupts axonal transport of SV components in photoreceptor R8 axons, but does not affect the transport of mitochondria. Genetic mosaic analysis, transgene rescue and cell-type-specific knockdown indicate that Bdl is required both presynaptically and postsynaptically for delivering SV components in R8 axons. Consistent with a role for Bdl in R8 axons, loss of $b d l$ causes a failure of R8-dependent phototaxis response to green light. $b d l$ interacts genetically with imac encoding for a member of the UNC-104/Imac/KIF1Afamily motor proteins, and is required for proper localization of Imac in R8 presynaptic terminals. Our results support a model in which Bdl mediates specific axo-dendritic interactions in a homophilic manner, which upregulates the Imac motor in promoting axonal transport of SV components for R8 presynaptic assembly and function.
\end{abstract}

Key words: axonal transport; borderless; color vision; Drosophila; motor; synaptic vesicle

Significance Statement

Whether and how synaptogenic adhesion at axo-dendritic contact sites regulates axonal transport of presynaptic components remain unknown. Here we show for the first time that a trans-synaptic adhesion molecule mediates specific interactions at axo-dendritic contact sites, which is required for upregulating the UNC-104/Imac/KIF1A motor in promoting axonal transport of synaptic-vesicle components for presynaptic assembly and function.

\section{Introduction}

After initial axon-dendrite contact, cellular morphogenetic events occur at both sides of the contact, which eventually leads to the formation of chemical synapses. Each synapse consists of specialized presynaptic and postsynaptic structures that allow

\footnotetext{
Received Jan. 10, 2019; revised May 15, 2019; accepted June 20, 2019.

Author contributions: H.S.S., S.A.C., and Y.R. designed research; H.S.S., S.A.C., and W.-T.C. performed research; H.S.S., S.A.C., W.-T.C., and Y.R. analyzed data; W.-T.C. contributed unpublished reagents/analytic tools; H.S.S. and Y.R. wrote the paper.

This work was supported by an operating Grant (MOP-14688 to Y.R.) from the Canadian Institutes of Health Research. We thank people in the Rao laboratory for comments and suggestions; Joe Larkin at McGill University Health Centre for assistance in the building and designing of the phototactic T-Maze apparatus; the Bloomington Stock Center and the Vienna Drosophila Resource Center for fly lines; T. Suzuki at Tokyo Institute of Technology for

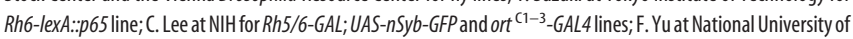
Singapore for UAS-imac-RFP; and T. Schwarz at Harvard for imac ${ }^{170}$, FRT42D and UAS-imac-RFP lines.

The authors declare no competing financial interests.

Correspondence should be addressed to Yong Rao at yong.rao@mcgill.ca.

https://doi.org/10.1523/JNEUROSCI.0075-19.2019

Copyright $\odot 2019$ the authors
}

proper neuronal communications. The assembly and function of presynaptic structures requires proper delivery of active-zone (AZ) and synaptic-vesicle (SV) components from soma to nerve terminals. For instance, axonal transport of piccolo-bassoon transport vesicles (PTVs) and synaptic vesicle precursors (SVPs) is required for the assembly of AZs and the accumulation of SVs for synapse formation and maintenance (Goldstein et al., 2008; Maeder et al., 2014). Whereas axonal transport of mitochondria is crucial for meeting energy demands at presynaptic terminals (Goldstein et al., 2008; Maeder et al., 2014).

Synapse formation involves rapid recruitment of SV and AZ components at the sites of axo-dendritic contact (McAllister, 2007; Chia et al., 2013). Accumulated evidence supports a key role for the kinesin-3 family motor Unc-104/Imac/KIF1A in regulating axonal transport of SVPs and PTVs (Hall and Hedgecock, 1991; Otsuka et al., 1991; Okada et al., 1995; Zhao et al., 2001; Pack-Chung et al., 2007; Barkus et al., 2008; Niwa et al., 2008). In the absence of Unc-104/Imac/KIF1A, most SVPs are unable to 

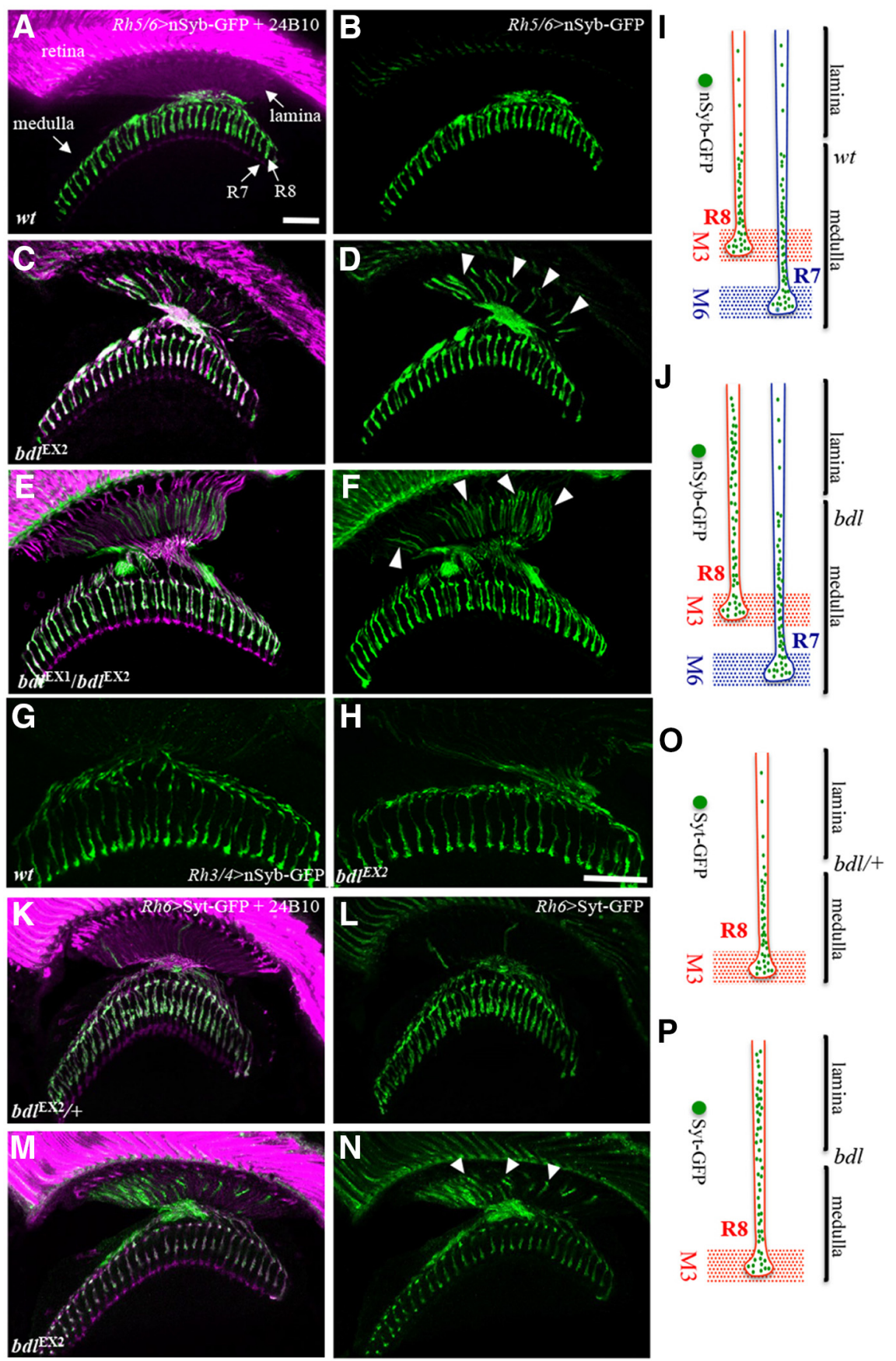

Figure 1. Many SV components were mislocalized to the proximal portion of R8 axon in $b d l$ mutants. $\boldsymbol{A}-\boldsymbol{D}$, Frozen sections of adult heads expressing the SV marker nSyb-GFP under control of the R8-specific driver Rh5/6-GAL4 (i.e., Rh5/6>nSyb-GFP), were stained with anti-GFP (green) and MAb24B10 (magenta). MAb24B10 recognizes the cell adhesion molecule Chaoptin expressed in all R-cell axons (Van Vactor et al., 1988). $\boldsymbol{A}$, In wild-type animals $(100 \%, n=7)$, nSyb-GFP staining was predominantly localized to $R 8$ axonal terminals in the medulla region. $\boldsymbol{B}$, The section in A was visualized with nSyb-GFP staining only. $\boldsymbol{C}$, In the majority of $b d l^{\mathrm{EX2}}$ homozygous mutant flies examined ( 6 of 7 animals), strong $n-S y b$ staining was also observed in the proximal portion of R8 axons in the lamina. $\boldsymbol{D}$, The section in $\mathbf{C}$ was visualized with nSyb-GFP staining only. Arrowheads indicate proximal portions of R8 axons with mislocalized nSyb-GFP.E, In all $b d l^{\mathrm{EX} 1} b d l^{\mathrm{EX} 2}$ transheterozygotes examined ( $n=6$ animals), strong n-Syb staining was observed in the proximal portion of R8 axons in the lamina. $\boldsymbol{F}$, The section in $\boldsymbol{E}$ was visualized with nSyb-GFP staining only. $\boldsymbol{G}, \boldsymbol{H}$, Frozen sections of adult heads expressing nSyb-GFP under control of the R7-specific driver Rh3/4-GAL4 (i.e., Rh3/4>nSyb-GFP), were stained with anti-GFP. G, In wild-type (100\%, $n=5$ animals), n-Syb staining was predominantly localized to R7 axonal terminals in the medulla region. $\boldsymbol{H}$, In all $\left.b d\right|^{\mathrm{EX2}}$ homozygous flies examined $(100 \%, n=5)$, $n$-Syb staining was still predominantly localized to $\mathrm{R} 7$ axonal terminals in the medulla region. $I, J$, Schematic illustrations showing the distribution of SV components in R7 and R8 axons in wild-type $(\boldsymbol{I})$ and $b d l$ mutants $(\boldsymbol{J})$. $\boldsymbol{K}-\boldsymbol{N}$, Frozen sections of adult heads expressing another SV marker Syt-GFP under control of the R8-specific driver Rh5/6-GAL4 (i.e., Rh5/6>Syt-GFP), were stained with anti-GFP (green) and MAb24B10 (magenta). $\boldsymbol{K},\left.\operatorname{In} b d\right|^{\mathrm{EX} 2} /+$ heterozygotes $(100 \%, n=5)$, Syt-GFP staining was predominantly localized to R8 axonal terminals in the medulla region. $\boldsymbol{L}$, , The section in $\boldsymbol{K}$ was visualized with Syt-GFP staining only. $\boldsymbol{M}$, In most $\left.b d\right|^{\mathrm{EX2}}$ homozygous mutants (11 of 13 animals), strong Syt-GFP staining was also observed in the proximal portion of R8 axons in the lamina. $\boldsymbol{N}$, The section in $\boldsymbol{K}$ was move from soma into axons. Recent studies also show that Unc-104/Imac/KIF1A is activated by the SVP-localized small arflike GTPase ARL-8 (Klassen et al., 2010; Niwa et al., 2017), and can also be modulated by the JNK MAP kinase pathway (Byrd et al., 2001; Wu et al., 2013). Although it is clear that synaptogenic signals such as trans-synaptic adhesion modulate local recruitments of SV components at the presynaptic terminals for the control of synaptic plasticity (Bury and Sabo, 2016), a recent in vitro study shows that neuroliginneurexin-mediated trans-synaptic adhesion does not significantly affect axonal transport of SV components in cultured cortical neurons (Bury and Sabo, 2014). It remains unknown whether and how synaptogenic adhesion at axo-dendritic contact sites regulates axonal transport of SV components to sites of presynaptic assembly in vivo.

In this study, we investigate the role of Borderless (Bdl) in the Drosophila visual system, which is an excellent model to study the mechanisms controlling neuronal circuit development and function (Sanes and Zipursky, 2010; Nériec and Desplan, 2016). Bdl belongs to the conserved IgSF9 subfamily of Ig superfamily (Hansen and Walmod, 2013). In our previous studies (Cameron et al., 2013; Chen et al., 2017), we show that Bdl functions as a cell adhesion molecule, and is capable of mediating both homophilic and heterophilic binding. Recent studies on IgSF9A and IgSF9B, homologs of Bdl in mammals, show that IgSF9A and IgSF9B function as trans-synaptic adhesion molecules by mediating homophilic binding (Hansen and Walmod, 2013; Woo et al., 2013). In Drosophila, Bdl is exclusively expressed in wrapping glia and is required for mediating axon-glia recognition at third-instar larval stage (Cameron et al., 2016). Whereas at later stages, $\mathrm{Bdl}$ is expressed in $\mathrm{R}$-cell axons, and negative regulation of Bdl by another Ig transmembrane protein Turtle is required for the tiling of R7 axonal terminals (Cameron et al., 2013). The role of Bdl in R-cell axons, however, remains unknown.

Our present study shows that loss of $b d l$ disrupted axonal transport of SV components in R8 axons, and caused the accumulation of the AZ protein Bruchpilot

visualized with Syt-GFP staining only. Arrowheads indicate proximal portions of R8 axons with mislocalized Syt-GFP. $\mathbf{O}, \boldsymbol{P}$, Schematic illustrations showing the distribution of SV components in R8 axons labeled with Syt-GFP in heterozygotes ( 0 ) and $b d l$ homozygous mutants (P). Scale bar, $20 \mu \mathrm{m}$. 
(Brp) in R8 soma. In contrast, axonal transport of the mitochondria remained normal in $b d l$ mutants. Cell-type-specific knockdown and transgene rescue support that Bdl is required both presynaptically and postsynaptically. Bdl interacts genetically with the Unc-104/Imac/KIF1A motor, and removing Bdl significantly decreased the levels of Imac in R8 axonal terminals. To our knowledge, our study shows for the first time that a transsynaptic cell adhesion molecule plays a specific role in regulating axonal transport of presynaptic components, and presents an excellent starting point for dissecting the signaling events that link synaptogenic adhesion and axonal transport of SV components for presynaptic assembly and function.

\section{Materials and Methods}

Genetics. imac ${ }^{170}$, FRT42D flies were provided by T. Schwarz at Harvard. UAS-imac-RFP flies were provided by F. Yu at National University of Singapore and T. Schwarz. Rh5/6-GAL; UAS-nSyb-GFP was provided by C. Lee at NIH. Rh6-lexA::p65 was provided by T. Suzuki at Tokyo Institute of Technology. ey-FLP; GMR-myr-mRFP, FRT40A/Cyo (BDSC\#7122), Rh3-GAL4 (BDSC\#7457), Rh4-GAL4 (BDSC\#8689 and \#8690), UAS-mito-GFP (BDSC\#8443), UAS-imac.GFP (BDSC\#6926), UAS- $\alpha$ Tub84B-GFP (BDSC\#7373), Rh5-brp.mCherry,Rh6-brp.mCherry (BDSC\#57322), lexAop-nSyb-spGFP1-10 (BDSC\#64315), and UAS-SytGFP (BDSC\#8443) flies were obtained for the Bloomington Drosophila Stock Center (BDSC). The UAS-bdl-RNAi line (VDRC\#4806) was obtained from Vienna Drosophila Resource Center (VDRC). For eyespecific mosaic analysis of Bdl in axonal transport of SV components, genetic crosses were performed to generate flies with the genotype $e y^{3.5}$ FLP; bdl ${ }^{\mathrm{EX} 2}$, FRT40A/ GAL80, FRT40A; Rh5/Rh6-GAL4, UAS-nSyb$\mathrm{GFP} /+$. To selectively remove $\mathrm{Bdl}$ in R7 and R1/R6 axons, genetic crosses were performed to generate flies with the genotype GMR-FLP; GMRmyr-mRFP, FRT40A/bdl ${ }^{\mathrm{EX} 2}$, FRT40A; Rh5/Rh6-GAL4, UAS-nSyb$G F P /+$. To examine the effects of removing $b d l$ on SV localization in R7 axons, genetic crosses were performed to generate flies with the genotype $b d l^{\mathrm{EX} 2} / b d l^{\mathrm{EX} 2} ; R h 3 / R h 4-G A L 4, U A S-n S y b-G F P /+$. For rescue experiments, genetic crosses were performed to generate flies with the genotype $b d l^{\mathrm{EX} 2} / b d l^{\mathrm{EX} 2}$; Rh5/Rh6-GAL4, UAS-nSyb-GFP/GMR-bdl, or $b d l^{\mathrm{EX} 2}$ / $b d l^{E X 2} ; R h 5 / R h 6-G A L 4, U A S-n S y b-G F P / H S-b d l$. For detecting endogenous Brp-mCherry puncta in R8 axons, genetic crosses were performed to generate flies with the genotypes $b d l^{\mathrm{EX} 2} / b d l^{\mathrm{EX} 2}$; Rh5-brp.mCherry, Rh6-brp.mCherry /+. For examining SV localization in R8 axons in which $b d l$ was knocked down in postsynaptic targets of R8, genetic crosses were performed to generate flies with the genotype ort ${ }^{\mathrm{C} 1-3}-G A L 4$; lexAop-nSybspGFP1-10/Rh6-lexA::p65; UAS-bdl-RNAi/+. For overexpressing imac in $\mathrm{R} 8$ axons in $b d l$ mutants, genetic crosses were performed to generate flies with the genotype $b d l^{\mathrm{EX} 2} / b d l^{\mathrm{EX} 2} ; R h 5 / R h 6-G A L 4, U A S-$ $n S y b$-GFP/UAS-imac-RFP. Previous studies show that the UAS-imacRFP transgene rescued the imac mutant phenotype (Pack-Chung et al., 2007; Zong et al., 2018).

Histology. Adult heads were dissected and fixed for $3 \mathrm{~h}$ on ice in $3.2 \%$ paraformaldehyde in phosphate buffer (PB), pH 7.2. Cryostat sections of adult and pupal heads were cut on a Leica CM3050 S or Leica CM1950 microtome at a thickness of $10 \mu \mathrm{m}$ and collected on Superfrost Plus slides (Fisher Scientific). Before the addition of primary antibodies, sections were blocked with $10 \%$ normal goat serum in PB with $0.5 \%$ Triton X-100 (PBT). Sections were then incubated with primary antibodies overnight at $4^{\circ} \mathrm{C}$. After washed $3 \times$ with PBT, sections were incubated with secondary antibodies for $45 \mathrm{~min}$. After washed $3 \times$ with PBT, $80 \mu \mathrm{l}$ of anti-fade gold was added to each slide, which was then covered with a glass coverslip and sealed with nail polish.

Antibodies were used at following dilutions: MAb24B10 (1:100; Developmental Studies Hybridoma Bank), rabbit polyclonal anti-GFP (1:1000; Invitrogen), rabbit polyclonal anti-Bdl (1:1000), chicken polyclonal anti-GFP for detecting spGFP1-10 (1:1000; Abcam), and chicken polyclonal anti-GFP (1:1000; Invitrogen). Secondary antibodies: antimouse AlexaFluor 647, anti-chicken AlexaFluor 488, anti-rabbit AlexaFluor 488, and (Invitrogen) were used at 1:750 dilution. Epifluorescent images were analyzed by confocal microscopy (Olympus, FluoView FV1000 LSM).

Quantification of relative fluorescence intensity. The Olympus FluoView or ImageJ software was used to measure fluorescent intensities in the proximal portion of $\mathrm{R} 8$ axons in the lamina and the distal portion of R8 axons in the medulla. Relative intensity of SV components in each region was calculated by normalizing the intensity of nSyb-GFP staining to that of MAb24B10 staining within the same region. Similarly, relative intensity of Imac.GFP in R8 axonal terminals was quantified by normalizing the intensity of Imac.GFP staining to that of MAb24B10 staining within the same region.

Molecular biology. For rescue experiments, the full-length $b d l$ coding sequence was amplified by PCR using the GH11322 EST clone as the template. $5^{\prime}$ primer CAATCGCGGCCGCATGCCAGCGAAACGCA and $3^{\prime}$ primer AGATCTGAGCAATCCTCAGGTGGAC were used. The resulting PCR products were subcloned into EcoRI and BglII sites of pGMR and pCaspeR-hs vectors. DNA constructs were verified by sequencing and used for generating transgenic lines.

Quantification of brp-GFP puncta in R8 soma. Confocal microscopy was used to acquire $1.0 \mu \mathrm{m}$ stacks of the samples. Brp-mCherry puncta were quantified using the FIJ ImageJ software. Particle Analyzer Tool was used to determine the size of puncta in the proximal region of $\mathrm{R} 8$ soma.

Phototactic T-maze behavioral assay. The behavioral assay was modified from that described previously (Yamaguchi et al., 2010). Flies were reared with $12 \mathrm{~h}$ light/dark cycles at constant humidity and temperature. Flies at the age of 7-10 d after eclosion were used in the experiments. For each genotype, $\sim 5-10$ trials were performed, and $\sim 50$ flies were tested in each trial. Flies were transported to behavior room at least $24 \mathrm{~h}$ before each experiment, and thus were able to habituate to the new environment.

Two light sources were used, including UltraFire WF-501B 375NM UV Ultra Violet LED Flashlight and Ultrafire WF-501B CREE XR-E G2 $150 \mathrm{~lm}$ Green LED Flashlight. "UV vs Green” choices were used to determine light preference. For each trial, flies were introduced into the T-Maze apparatus and allowed to habituate for $60 \mathrm{~s}$. The lights were then turned on, and flies were introduced to the choice point for $20 \mathrm{~s}$. Flies moved into either the Green or UV zone, or did not move out of the choice point (neutral). Flies were then anesthetized with $\mathrm{CO}_{2}$ and counted. Light preference index (PI) was quantified as follows:

$$
\begin{aligned}
& \left(\left(\frac{\text { number of flies in blue or green zone }- \text { Number of flies in UV zone }}{\text { number of flies in blue or green zone }+ \text { Number of flies in UV zone }}\right)\right. \\
& \left.\quad \times\left(1-\frac{\text { number of flies in neutral zone }}{\text { number of total flies }}\right)\right) .
\end{aligned}
$$

Because fly behaviors are sensitive to variations in experimental conditions, this may cause behavioral variations even within the control groups from one experiment to another experiment. To minimize the effects of variations in experimental conditions in each experiment, control and experimental flies for behavioral comparison were generated and collected at same time period, and phototactic assays were performed on the same day.

Statistical analysis. For experiments involving the comparison of two groups, statistical analysis was performed using two-tailed $t$ tests. For experiments involving the comparison of more than two groups, statistical analysis was performed using one-way ANOVA followed by post hoc Tukey's test. The difference is considered as significant when a $p$ value is $<0.05$.

\section{Results}

\section{Loss of $b d l$ disrupted axonal transport of SV components in R8 axons}

In our previous study, we show that $\mathrm{Bdl}$ is expressed in both $\mathrm{R} 7$ and $\mathrm{R} 8$ axons, and is also present in the optic lobe (Cameron et al., 2013). However, R-cell axon guidance and layer-specific target selection remained normal in bdl-null mutants (Cameron et al., 2013). To test whether Bdl plays a role in the regulation of $\mathrm{R}$-cell presynaptic assembly and function, we used a SV-specific marker neuronal synaptobrevin-GFP (nSyb-GFP; Estes et al., 
2000), to examine R7 and R8 presynaptic development. R8 photoreceptors express rhodopsin (Rh) Rh5 or Rh6, and show preference for blue and green light (Vasiliauskas et al., 2011). Whereas R7 photoreceptors express Rh3 or Rh4, and show preference for light in the UV spectrum (Vasiliauskas et al., 2011). R7 and R8 axons from a single ommatidia project through the lamina into the deeper medulla. R8 axons form synapses specifically within M1-M3 sublayers of the medulla. R7 axons defasciculate from R8 axons at M3 and project deeper in the medulla, where they establish synapses in the M4-M6 sublayers.

SV components in R7 and R8 axons were labeled by expression of nSyb-GFP under control of R7-specific drivers $R h 3$ GAL4 and Rh4-GAL4 (i.e., Rh3/Rh4GAL4) and R8-specific drivers Rh5-GAL4 and Rh6-GAL4 (i.e., Rh5/Rh6-GAL4; Fig. $1)$, respectively. In wild-type, SV components in both R7 and R8 axons were predominantly targeted to the presynaptic terminals within the medulla (Fig. $1 A$, $B, G)$. Surprisingly, we found that many SV components were mislocalized to the proximal portion of $\mathrm{R} 8$ axons within the lamina in $b d l$ mutants (Fig. $1 C-F, I$ ). In contrast, the localization of SV components remained normal in $\mathrm{R} 7$ axons in $b d l$ mutants (Fig. $1 H, J$ ).

To further confirm the SV phenotype in R8 axons, we used a different SV marker synaptotagmin-GFP (Syt-GFP; Zhang et al., 2002), to examine the targeting of SV components. Consistently, we found that many Syt-GFP-positive vesicles were abnormally localized to the proximal portion of $\mathrm{R} 8$ axons within the lamina in $b d l$ mutants (Fig. $1 M, N, P$ ).

$b d l$ is required cell-autonomously in $\mathrm{R} 8$ axons for the targeting of

SV components

Above phenotypes in $b d l$ mutants may reflect a cell-autonomous role for Bdl in R8 axons, or a non-cell-autonomous role in its postsynaptic targets. To distinguish between these possibilities, we performed genetic mosaic analysis. Homozygous $b d l$ mutant R-cell clones were generated in the eye by performing eye-specific mitotic recombination. We found that specific removal of $b d l$ in $\mathrm{R}$-cell axons, but not in the optic lobe, caused a SV mistargeting phenotype identical to that in $b d l$ mutants (Fig. $2 A, B$ ), indicating that $b d l$ is required in R-cell axons for axonal transport of SV components.

Because eye-specific mitotic recombination removed $b d l$ in both $\mathrm{R} 7$ and $\mathrm{R} 8$
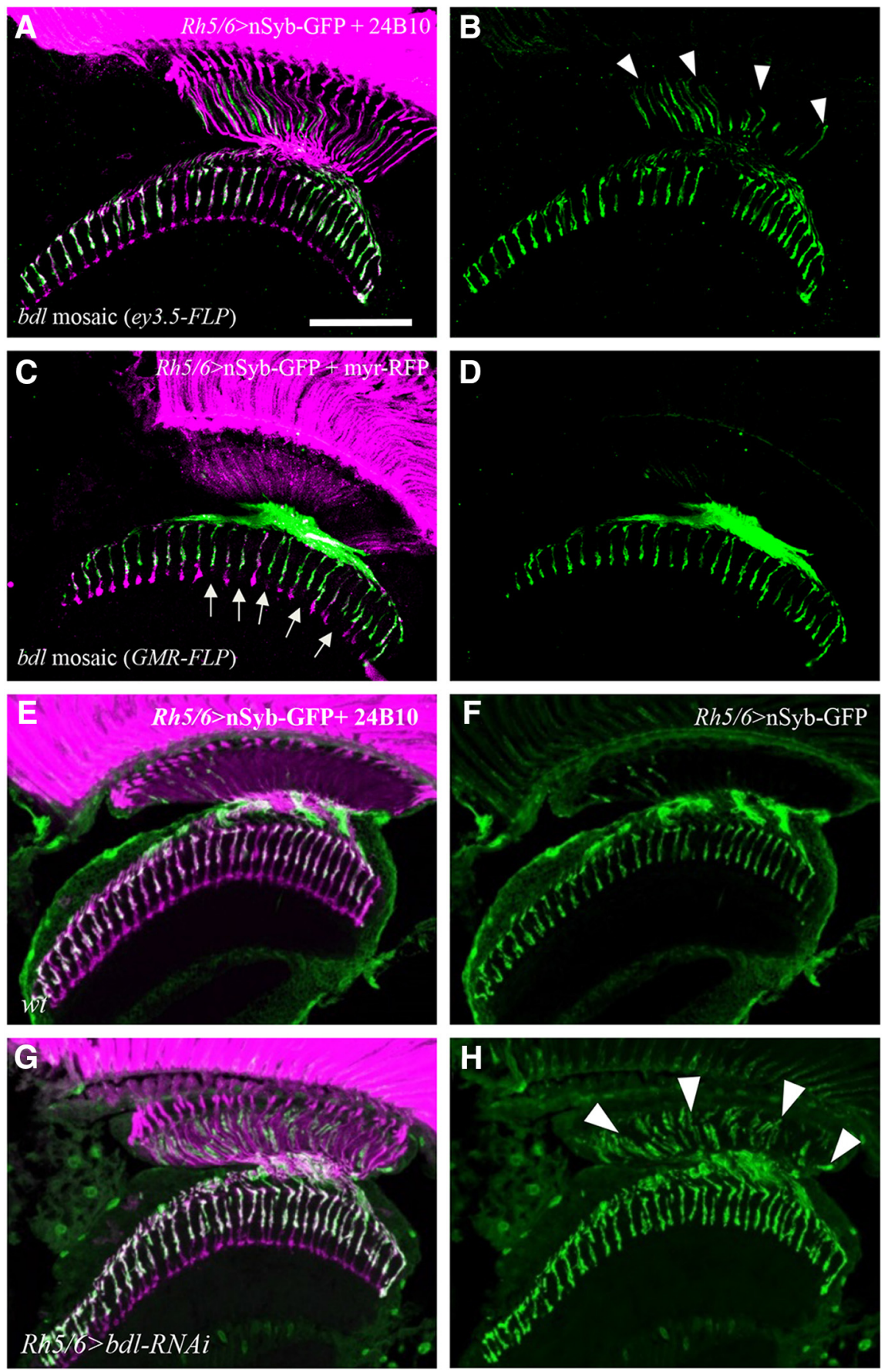

Figure 2. $\quad b d$ is required cell-autonomously in R8 axons. $A, B$, Frozen sections of adult heads were stained with anti-GFP (green) and MAb24B10 (magenta). $A$, In all eye-specific $b d l$ mosaic animals examined (100\%, $n=6$ animals), strong nSyb-GFP staining was observed in the proximal portion of $b d l$ mutant $R 8$ axons. $\boldsymbol{B}$, The section in A was visualized with nSyb-GFP staining only. Arrowheads indicate proximal portions of R8 axons with mislocalized nSyb-GFP. C, D, Frozen sections of adult heads were stained with anti-GFP (green) and anti-RFP (magenta). Homozygous $b d l$ mutant R7 axons were generated by GMR-FLP-induced mitotic recombination. C, In all GMR-FLP-induced $b d l$ mosaic animals examined (100\%, $n=6$ animals), nSyb-GFP staining (green) was still predominantly localized to R8 axonal terminals in the medulla. Wild-type or heterozygous R-cell axons were labeled with GMR-myr-mRFP (magenta). Mosaic columns were identified by the absence of RFP staining in $b d /$ mutant R7 axons (arrows). D, The section in $\boldsymbol{C}$ was visualized with nSyb-GFP staining only. $\boldsymbol{E}$, In wild-type double-stained with anti-GFP (green) and MAb24B10 (magenta), nSyb-GFP staining was predominantly localized to R8 axonal terminals in the medulla ( $100 \%, n=5$ animals). $\boldsymbol{F}$, The section in $\boldsymbol{E}$ was visualized with nSyb-GFP staining only. $\boldsymbol{G}$, In flies expressing a UAS-bdl-RNAi transgene under control of the R8-specific driver Rh5/6-GAL4, strong nSyb-GFP staining was also observed in the proximal portion of R8 axons in the lamina (5 of 6 animals). $\boldsymbol{H}$, The section in $\boldsymbol{G}$ was visualized with $n$ Syb-GFP staining only. Arrowheads indicate proximal portions of R8 axons with mislocalized nSyb-GFP. Scale bar, $20 \mu \mathrm{m}$. 

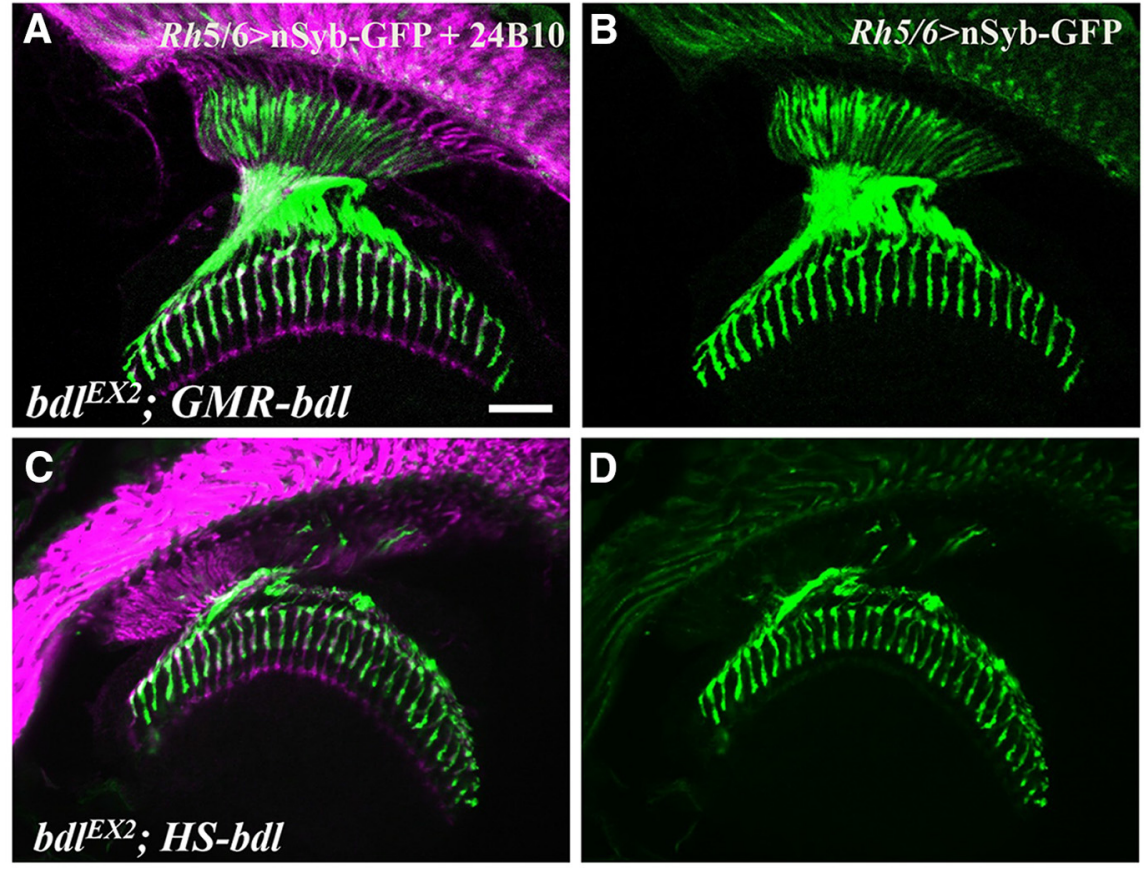

E

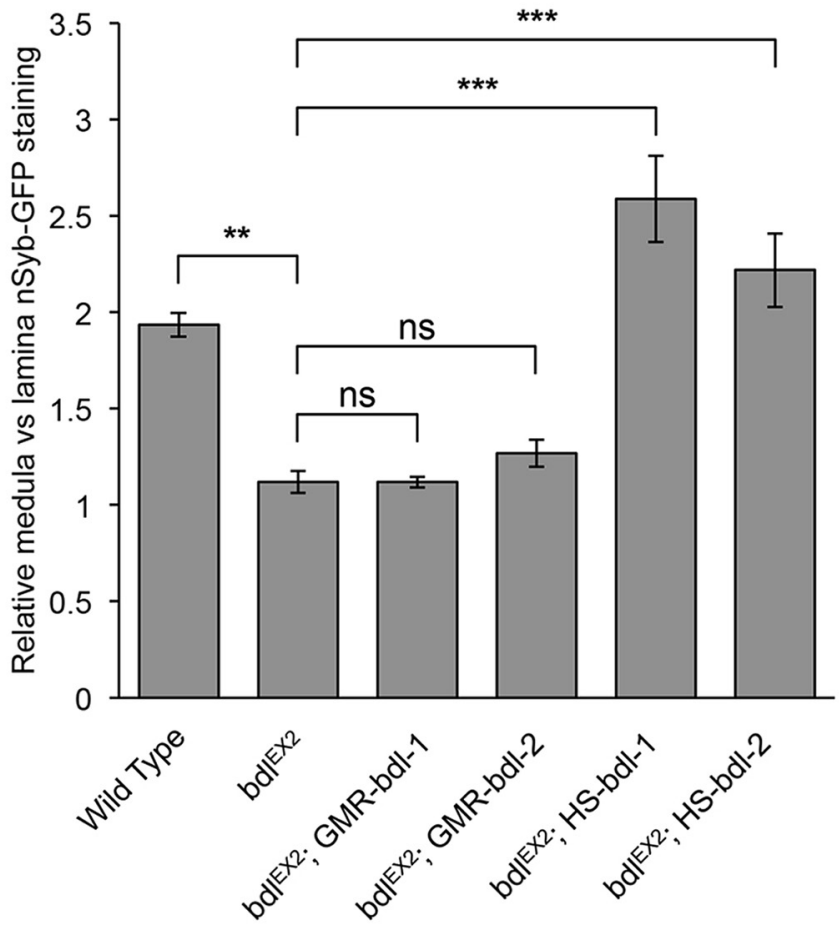

Figure 3. Expression of $b d$ l in R-cell axons was not sufficient for rescuing the $S V$ phenotype in $b d l$ mutants. $A-D$, Frozen sections of adult heads expressing nSyb-GFP under control of the R8-specific driver Rh5/6-GAL4, were stained with anti-GFP (green) and MAb24B10 (magenta). $A$, Restoring $b d l$ expression in all R-cell axons under control of the eye-specific GMR promoter did not rescue the SV phenotype, as many SV components were still mislocalized to the proximal portion of R8 axons in the lamina. Genotype: $b d{ }^{\mathrm{EX2}} ;$ GMR-bdl/ Rh5/6-GAL4, UAS-nSyb-GFP. Two independent GMR-bdl transgenic lines were used in the experiments. Eight individuals were examined in each experiment. $\boldsymbol{B}$, The section in $\boldsymbol{A}$ was visualized with nSyb-GFP staining only. $\boldsymbol{C}$, Restoring $b d l$ expression in both R-cell axons and the optic lobe under control of the heat-inducible promoter completely rescued the SV phenotype. Genotype: $b d l^{\mathrm{EX}} ;$; HS-bdl/ Rh5/6-GAL4, UAS-nSyb-GFP. Two independent HS-bdl transgenic lines were used in the experiments. At least five individuals were examined in each experiment. $\boldsymbol{D}$, The section in $\mathbf{C}$ was visualized with nSyb-GFP staining only. $\boldsymbol{E}$, The ratio of medulla versus lamina relative nSyb-GFP staining intensities was quantified. One-way ANOVA followed by post hoc Tukey's test. ${ }^{* *} p<0.01,{ }^{* * *} p<0.001 ; n \mathrm{n}, p>0.05$. Error bars indicate SEM. Scale bar, $20 \mu \mathrm{m}$. move $b d l$ in $\mathrm{R} 7$ but not in R8 axons. We found that the localization of SV components remained normal in wild-type $\mathrm{R} 8$ axons when $b d l$ was removed in $\mathrm{R} 7$ axons within the same column (Fig. 2C,D). This result argues against a non-cell-autonomous role for Bdl in $\mathrm{R} 7$ axons in regulating SV targeting in $\mathrm{R} 8$ axons within the same column.

To further confirm that Bdl plays a cell-autonomous role in $\mathrm{R} 8$ axons, we performed R8-specific knockdown experiments. A UAS-bdl-RNAi transgene that has been shown previously to knock down $b d l$ effectively (Cameron et al., 2016), was expressed specifically in $\mathrm{R} 8$ axons under control of the R8-specific driver Rh5/Rh6GAL4. A similar SV phenotype was observed when $b d l$ was specifically knocked down in R8 axons (Fig. 2G,H).

In summary, above results from genetic mosaic analysis and R8-specific knockdown experiments suggest strongly that Bdl plays a cell-autonomous role in R8 axons for axonal transport of SV components.

\section{$b d l$ is required both presynaptically} and postsynaptically

Above results support a necessary role for $\mathrm{Bdl}$ in R8 axons. To determine whether the expression of Bdl in R8 axons is sufficient, we performed transgene rescue experiments. A transgene in which the complete $b d l$ cDNA is located downstream of the eye-specific promoter $G M R$ was introduced into bdl-null mutants. However, we found that the SV phenotype in $b d l$ mutants could not be rescued by restoring $b d l$ expression in $\mathrm{R}$-cell axons (Fig. $3 A, B, E$ ). This result raises the possibility that $\mathrm{Bdl}$ is required in both $\mathrm{R} 8$ axons and their targets in the medulla.

To test this, we examined the effects of restoring $b d l$ in both $\mathrm{R}$-cell axons and the optic lobe on the SV phenotype by introducing a $b d l$ transgene containing the $b d l$ coding sequence downstream of a heat-inducible promoter into $b d l \mathrm{mu}-$ tants. Interestingly, we found that expression of $b d l$ in both R-cell axons and the target region completely rescued the SV phenotype in $b d l$ mutants (Fig. $3 C-E)$.

Together, our results suggest that Bdl is required both presynaptically and postsynaptically for axonal transport of SV components. axons that associate with each other closely within the medulla, it remained possible that the SV phenotype in $\mathrm{R} 8$ axons was caused by loss of $b d l$ in $\mathrm{R} 7$ axons. To address this possibility, mitotic recombination under control of the GMR-FLP was used to re-
Loss of $b d l$ caused the accumulation of the AZ protein Brp in the proximal region of $\mathrm{R} 8$ soma

In addition to axonal transport of SV components, presynaptic assembly and function also requires the recruitment of AZ com- 

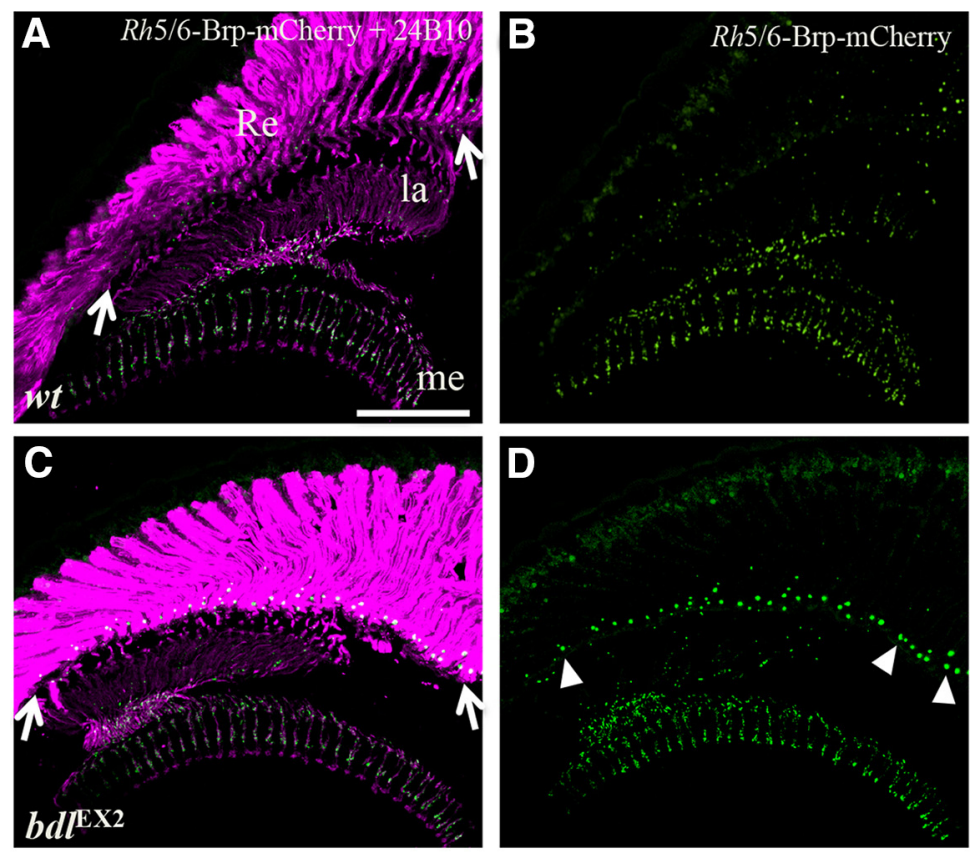

\section{E}
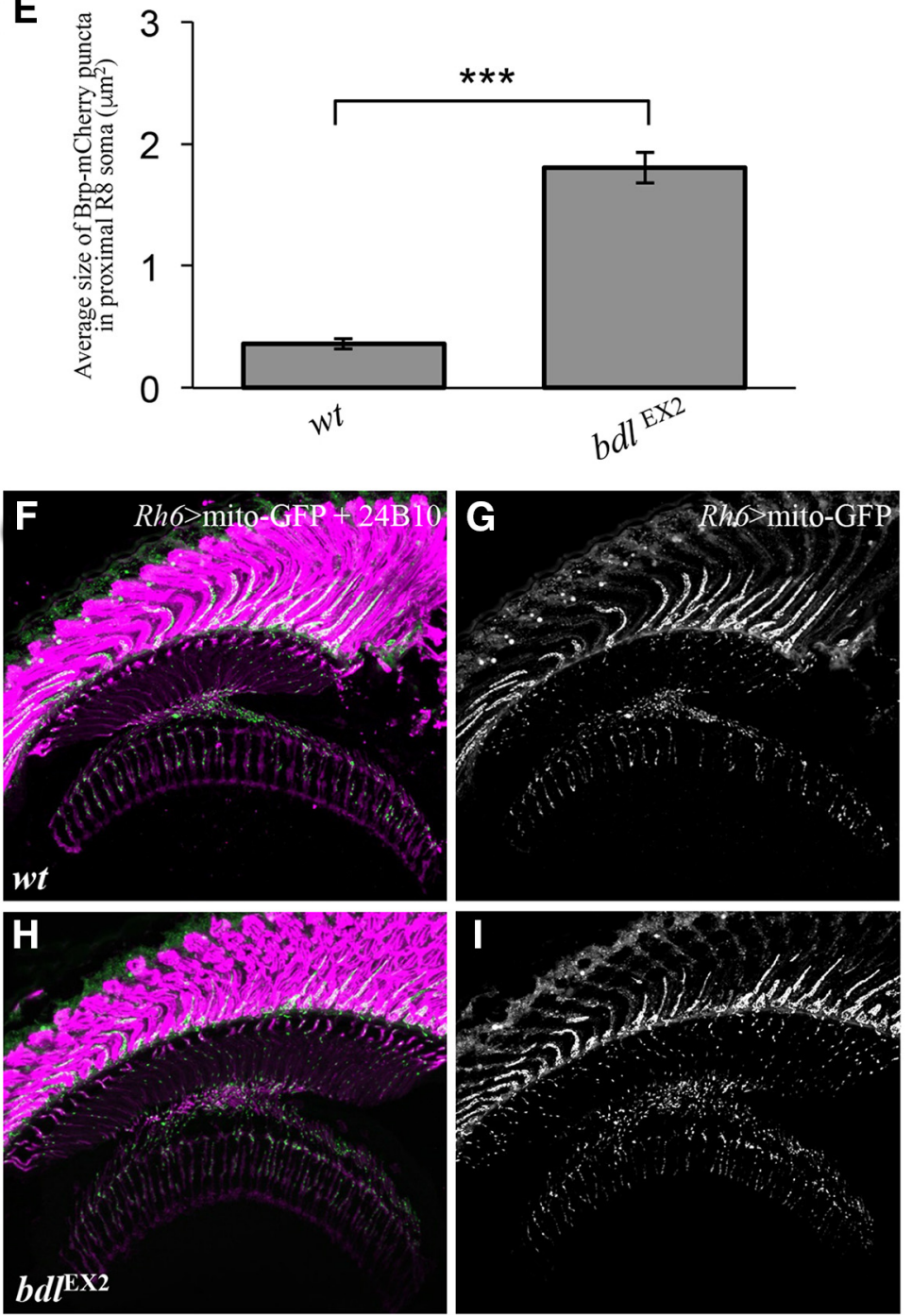

Figure 4. Loss of $b d l$ affected the transport of the AZ protein Brp but not the transport of mitochondria in R8 axons. $A-D$, Frozen sections of adult heads carrying Rh5/6-Brp-mCherry were double-stained with anti-GFP (green) and MAb24B10 (magenta). $\boldsymbol{A}$, In wild-type animals $(100 \%, n=6)$, Brp-mCherry puncta was predominantly localized to $\mathrm{R} 8$ axonal terminals in the medulla region. ponents and mitochondria. To test whether Bdl also plays a role in axonal transport of AZ components, we examined the potential effects of $b d l$ mutations on the targeting of the AZ protein Brp. Brp is a homologous to human $\mathrm{AZ}$ protein ELKS/CAST, and is essential for the establishment and maintenance of active zones for synapse formation and function in Drosophila (Wagh et al., 2006).

The distribution of Brp in R8 was visualized using the R8-specific AZ marker Rh5/Rh6-Brp-mCherry, which labels AZs in R8 axonal terminals and PTVs that transport AZ components in R8 (Ting et al., 2014). In wild-type (Fig. 4A, B), BrpmCherry punta were predominantly localized to R8 axonal terminals in the medulla region, where R8 axons form synaptic connections with their target neurons. Interestingly, we found that in all $b d l$ homozygous mutants examined $(n=10$; Fig. $4 C-E)$, abnormal large BrpmCherry particles were accumulated at the proximal region of $\mathrm{R} 8$ soma in the retina, which is close to the axonal initial segment. This phenotype was never observed in wild-type animals $(n=6$; Fig. 4 , compare $B, D)$. This result suggests that $\mathrm{Bdl}$ may also be required for the transport of Brp from R8 soma into the axon.

\section{Loss of $b d l$ did not affect axonal transport of mitochondria}

We then examined whether loss of $b d l$ affects axonal transport of mitochondria. The distribution of mitochondria in R8 axons was visualized using the mitochondria marker UAS-Mito-GFP under control of the R8-specific driver Rh5/Rh6-GAL4. However, no obvious difference in the local-

\section{$\leftarrow$}

$\boldsymbol{B}$, The section in $\boldsymbol{A}$ was visualized with Brp-mCherry staining only. C, In bdl ${ }^{\mathrm{EX} 2}$ homozygous mutants (100\%, $\left.n=10\right)$, abnormal large Brp-mCherry particles were accumulated at the proximal region (arrows) of R8 soma in the retina. $D$, The section in C was visualized with Brp-mCherry staining only. Arrowheads indicate abnormal large Brp-mCherry particles. $\boldsymbol{E}$, The size of Brp-mCherry puncta in the proximal region of R8 soma was quantified. Compared with that in wild-type, the size of Brp puncta in the proximal region of R8 soma in bdl mutants showed a significant increase. Student's $t$ test, ${ }^{* * *} p=4.5 \mathrm{e}-07$. Error Bars indicate SEM. F-I, Frozen sections of adult heads expressing UAS-mito-GFP under control of the R8-specific driver Rh6-GAL4, were stained with anti-GFP (green) and MAb24B10 (magenta). $\boldsymbol{F}$, In wild-type (100\%, $n=7$ animals), mitochondria were detected in both proximal portions of $R 8$ axons in the lamina and R8 axonal terminals in the medulla. $\boldsymbol{G}$, The section in $\boldsymbol{F}$ was visualized with mito-GFP staining only. $\boldsymbol{H},\left.\ln b d\right|^{\mathrm{EX} 2}$ mutants $(100 \%, n=6$ animals), the pattern of mitochondria distribution was similar to that in wild-type. $\boldsymbol{I}$, The section in $\boldsymbol{H}$ was visualized with mito-GFP staining only. Scale bar, $20 \mu \mathrm{m}$. 
A
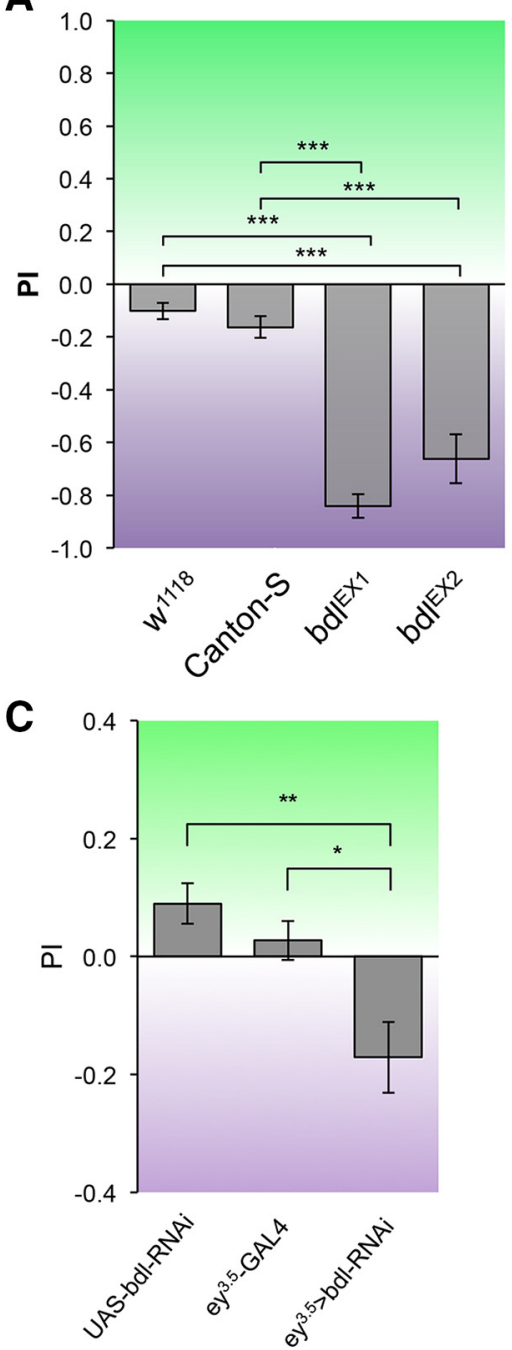

B

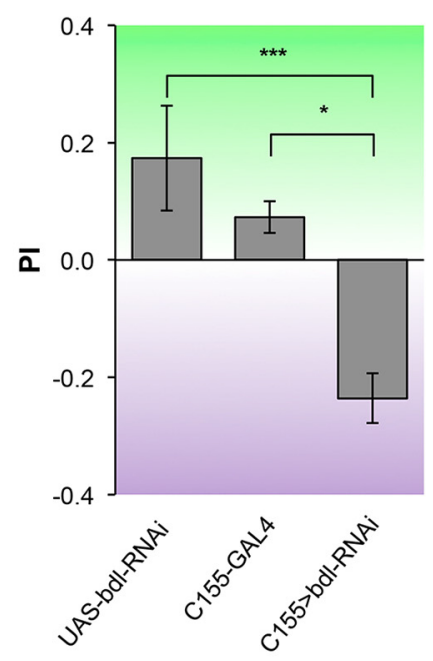

D

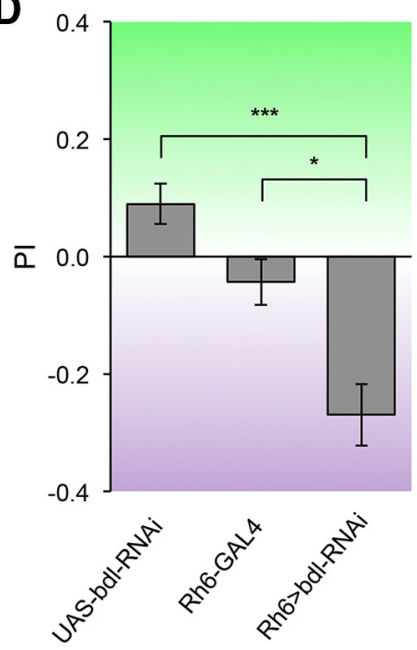

Figure 5. R8-dependent phototaxis response was disrupted in bd/ mutants. Flies were given UV vs green choice. Light PI was calculated as described in Materials and Methods. $\boldsymbol{A}$, Canton- $\boldsymbol{S}$ wild-type and $w^{1118}$ flies could be attracted to both UV and green light sources. However, both $b d l^{\mathrm{EX} 1}$ and $b d l^{\mathrm{EX} 2}$ homozygous mutant flies were predominantly attracted toward UV light source. $\boldsymbol{B}$, bdl was knocked down in flies carrying a pan-neuronal-specific driver C155-GAL4 and a UAS-bdl-RNAi transgene. Compared with control flies that carried C155-GAL4 or UAS-bdI-RNAi only, flies carrying both C155-GAL4 and UAS-bdl-RNAi showed a much greater preference for UV light. C, Eye-specific knockdown of $b d l$ was performed by expressing UAS-bdl-RNAi under control of the eyespecific driver $e y^{3.5}$-GAL4. Reducing $b d l$ in the eye significantly decreased the preference for green light. $\boldsymbol{D}, b d l$ was specifically knocked down in green-sensitive R8 photoreceptors (i.e., R8y) by expressing UAS-bdl-RNAi under control of the R8y-specific driver Rh6-GAL4. Knocking down bdl in green-sensitive R8 decreased the preference for green light. For each genotype, $\sim 5-10$ trials were performed, and $\sim 50$ flies were tested in each trial. One-way ANOVA followed by post hoc Tukey's test. ${ }^{*} p<0.05,{ }^{* *} p<$ $0.01,{ }^{* * *} p<0.001$. Error bars indicate SEM.

ization of mitochondria in $\mathrm{R} 8$ axons between wild-type and $b \mathrm{dl} \mathrm{mu}-$ tants was observed (Fig. 4, compare $H, I$ to $F, G$ ).

\section{Loss of $b d l$ disrupted R8-dependent phototaxis response}

We then performed differential phototaxis experiments to examine whether loss of $b d l$ affects R8 function. Among photoreceptors, R7 expresses $\mathrm{Rh} 3 / \mathrm{Rh} 3$ and is $\mathrm{UV}$-sensitive. Approximately $70 \%$ of R8 (i.e., R8y) express Rh6 and are green-sensitive, whereas $\sim 30 \%$ of R8 (i.e., R8p) express Rh5 and are blue-sensitive. Previous studies show that manipulating the functions of R7 or R8 could switch fly light preference (Yamaguchi et al., 2010).

A T-maze apparatus was used to examine the ability of flies to distinguish lights at different wavelength similarly as described previously (Yamaguchi et al., 2010). Flies were given the UV vs green choice to test their light preference. When given UV vs green choice, wild-type flies did not show a significant difference in their preference for different lights (Fig. 5A). However, we found that in the absence of $b d l$, flies largely ignored green light source, and were predominantly attracted toward UV light source (Fig. 5A).

We then performed cell-type-specific knockdown experiments to determine whether $\mathrm{Bdl}$ is required cell-autonomously for R8 function. $b d l$ was knocked down by expressing a UAS-bdl-RNAi transgene under control of pan-neuronal driver C155-GAL4 (Fig. 5B), eye-specific driver $e y^{3.5}-G A L 4$ (Fig. 5C), or greensensitive-R8-specific driver Rh6-GAL4 (Fig. 5D). Compared with control flies carrying GAL4 driver or UAS-bdl-RNAi only, flies carrying both GAL4 driver and $U A S-b d l-R N A i$ showed a significant decrease in the preference for green light source (Fig. 5B-D).

Together, above results support an essential and cell-autonomous role for $\mathrm{Bdl}$ in the control of R8 function.

\section{Knocking down $b d l$ in postsynaptic target neurons in the optic lobe caused the mislocalization of SVs in R8 axons} The results from transgene rescue (Fig. 3) raise the interesting possibility that $\mathrm{Bdl}$ is required in both $\mathrm{R} 8$ and its postsynaptic target neuron for the transport of SV components in R8 axons. To further address this, we examined whether reducing $\mathrm{Bdl}$ in postsynaptic target neurons in the optic lobe affects the localization of SV components in $\mathrm{R} 8$ axons.

To knock down $b d l$ in postsynaptic targets of R8, ort ${ }^{\mathrm{Cl}-3}$-GAL4 (Gao et al., 2008) was used to drive the expression of UAS- $b d l$ $R N A i$. The lexA/lexAop system was used to label SV components in bdl knockdown flies; the SV marker lexAop-nSybspGFP1-10 (Macpherson et al., 2015), was expressed under control of the greensensitive-R8-specific driver Rh6-lexA::p65 (Berger-Müller et al., 2013). We found that knocking down $b d l$ in postsynaptic targets of R8 caused a similar SV mislocalization phenotype in all individuals examined $(100 \%, n=4$ animals; (Fig. 6C,D).

\section{Knocking down $b d l$ in postsynaptic target neurons in the} optic lobe disrupted R8-dependent phototaxis response We then examined whether knocking down $b d l$ in postsynaptic target neurons affects R8-dependent phototaxis response. $b d l$ was knocked down in postsynaptic targets of R8 by expressing UAS$b d l-R N A i$ under control of the ort ${ }^{\mathrm{C} 1-3}-G A L 4$ driver. Flies were then given UV vs green choice to examine their light preference. Compared with control flies carrying ort ${ }^{\mathrm{Cl}-3}$-GAL4 or UAS-bdl$R N A i$ only, $b d l$ knockdown flies showed a significant decrease in the preference for green light source (Fig. 6E). 


\section{bdl interacted genetically with imac}

To further understand the action of Bdl in regulating axonal transport of SV components, we examined the potential effects of $b d l$ mutations on the organization of microtubules in R8 axons. Microtubules were labeled with the marker UAS-GFP- $\alpha$ Tub84B under control of the R8-specific driver Rh6-GAL4. Similarly as described previously (Sugie et al., 2015), we quantified the percentage of organized microtubule threads in each R8 axonal terminal. However, no obvious defect was observed (data not shown).

Previous studies show that the fly Imac motor protein and its orthologs Unc-104 in Caenorhabditis elegans and KIF1A in mammals play an essential role in regulating axonal transport of SV components (Hall and Hedgecock, 1991; Otsuka et al., 1991; Okada et al., 1995; Zhao et al., 2001; Pack-Chung et al., 2007; Barkus et al., 2008; Niwa et al., 2008). Consistently with previous reports (Pack-Chung et al., 2007), we showed that the removal of Imac in R-cell axons severely disrupted axonal transport of SV components, as the majority of SV components were localized abnormally in $\mathrm{R} 8$ cell bodies in the retina when imac was removed in $\mathrm{R}$ cells by eye-specific mitotic recombination (Fig. 7C-E).

To test the potential genetic interaction between $b d l$ and imac, we performed epistasis analysis. Most $b d l /+$ heterozygotes ( $\sim 93 \%, n=15$; Fig. $7 F, I)$ and imac/+ heterozygotes $(\sim 87 \%$, $n=16$; Fig. $7 G, J)$ displayed normal SV localization pattern in R8 axons. Interestingly, when flies were transheterozygous for $b d l$ and imac (i.e., $b d l / i m a c)$, most individuals showed a $b d l$-like SV mislocalization phenotype $(\sim 80 \%, n=15$; Fig. $7 \mathrm{H}, \mathrm{K})$. This synergistic phenotype suggests that $\mathrm{Bdl}$ may regulate axonal transport of SV components by modulating Imac.

\section{The levels of the Imac motor were significantly reduced in R8} presynaptic terminals

To further understand the action of Bdl in the regulation of Imac, we examined whether reducing $b d l$ affects the levels and distribution of Imac. The distribution of Imac in R8 axons was visualized with the Imac.GFP marker, which has been shown previously to recapitulate the localization of endogenous Imac (Barkus et al., 2008). Rh6-GAL4 was used to drive the expression of UASimac.GFP in green-sensitive R8 axons of y-type ommatidia (Tahayato et al., 2003). In wild-type, $\sim 80 \%$ R8 axons were stained with imac.GFP (Fig. $8 A-C, G$ ), consistent with that Rh6 is only expressed in green-sensitive R8y photoreceptor cells (Salcedo et al., 1999; Yamaguchi et al., 2010). We then performed cell-type-specific knockdown to reduce the level of Bdl in R8 axons. Interestingly, we found that compared with that of control flies carrying Rh6-GAL4 and UAS-imac.GFP (Fig. 8A-C) or control flies carrying Rh6-GAL4, UAS-imac.GFP and UAS-CD2.HRP (Fig. 8G), bdl knockdown in flies carrying Rh6-GAL4, UASimac.GFP and UAS-bdl-RNAi significantly decreased the percentage of $\mathrm{R} 8$ axons in which Imac.GFP was localized to R8 terminals (Fig. $8 D-G$ ). We also quantified relative levels of Imac.GFP in R8 axonal terminals. When $b d l$ was knocked down, the levels of Imac.GFP staining in Imac.GFP-positive R8 presynaptic terminals were also significantly reduced (Fig. $8 D-F, H$ ).

\section{Discussion}

Our present study identifies for the first time a cell adhesion molecule that plays a novel and specific role in promoting axonal transport of SV components for presynaptic assembly and function. Loss of $b d l$ selectively disrupted axonal transport of SV components in R8 axons, and also caused the accumulation of the AZ protein Brp in the proximal region of $\mathrm{R} 8$ soma. In contrast, $b d l$ mutations did not affect axonal transport of mitochondria. Ge-
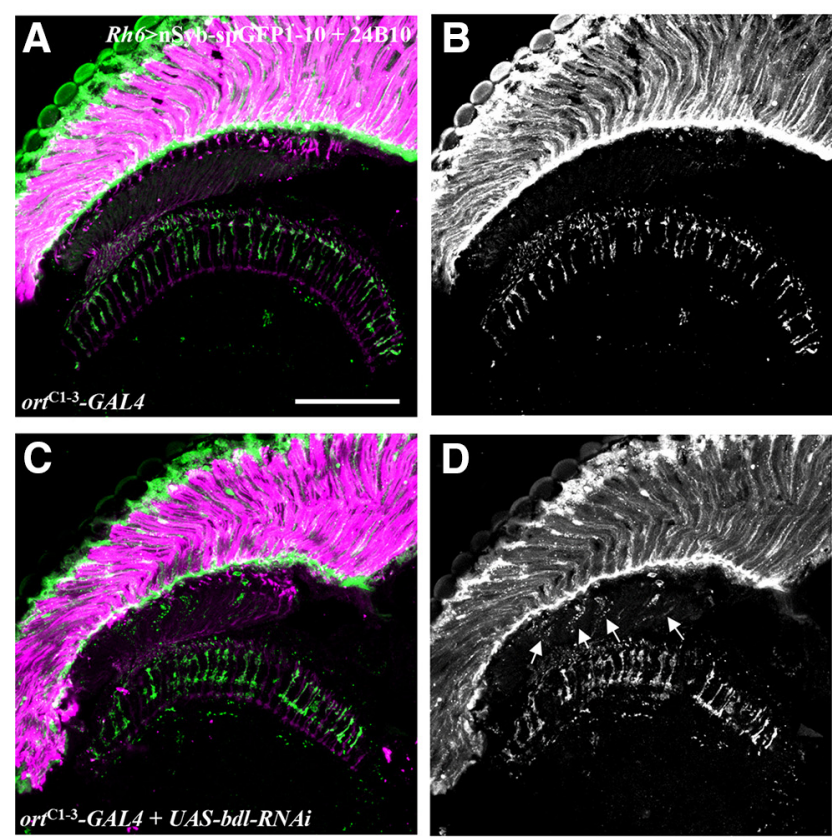

E

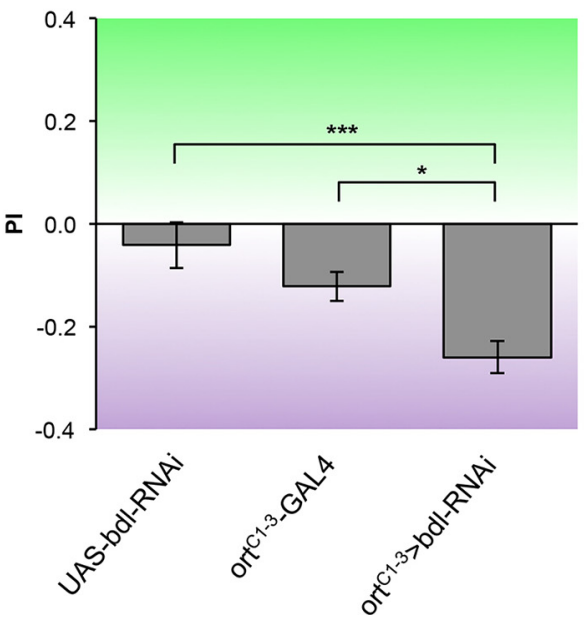

Figure 6. Knockdown of $b d l$ in postsynaptic target neurons in the optic lobe disrupted the transport of SVs in R8 axons and R8-dependent phototaxis response. $\boldsymbol{A}-\boldsymbol{D}$, Frozen sections of adult heads expressing the SV marker lexAop-nSyb-spGFP1-10 under control of the greensensitive-R8-specific driver Rh6-lexA::p65, were stained with anti-GFP (green) and MAb24B10 (magenta). $\boldsymbol{A}$, In most control animals carrying ort ${ }^{\mathrm{C1}-3}$-GAL4 only (6 of 7 animals), SVs labeled with nSyb-spGFP1-10 were predominantly localized to R8 axonal terminals in the medulla region. $\boldsymbol{B}$, The section in $\boldsymbol{A}$ was visualized with $n S y b-5 p G F P 1-10$ staining only. $\boldsymbol{C}$, When $b d /$ was knocked down in postsynaptic target neurons in the optic lobe by expressing UAS-bdl-RNAi under control of the ort ${ }^{\mathrm{C} 1-3}$-GAL4 driver, strong nSyb-spGFP1-10 staining was also observed in the proximal portion of $R 8$ axons in the lamina (100\%, $n=4$ animals). $\boldsymbol{D}$, The section in $\boldsymbol{C}$ was visualized with nSyb-spGFP1-10 staining only. Arrowheads indicate proximal portions of R8 axons with mislocalized nSyb-spGFP1-10. $\boldsymbol{E}$, Flies were given UV vs green choice. Knocking down $b d l$ in postsynaptic targets of R8 by expressing UAS-bdl-RNAi under control of ort ${ }^{\mathrm{C} 1-3}$. $G A L 4$, significantly decreased the preference for green light. For each genotype, $\sim 10-13$ trials were performed, and $\sim 50$ flies were tested in each trial. One-way ANOVA followed by post hoc Tukey's test. ${ }^{*} p<0.05,{ }^{* * *} p<0.001$. Error bars indicate SEM. Scale bar, $20 \mu \mathrm{m}$.

netic mosaic analysis, transgene rescue and cell-type-specific knockdown indicate that $\mathrm{Bdl}$ is required both presynaptically and postsynaptically. Removing $b d l$ also disrupted R8-dependent phototaxis response, consistent with a role for $\mathrm{Bdl}$ in the control of R8 function. We also show that $b d l$ interacted genetically with $i m a c$, and $b d l$ knockdown significantly decreased the levels of the Imac motor in R8 axonal terminals. Our results support a model 

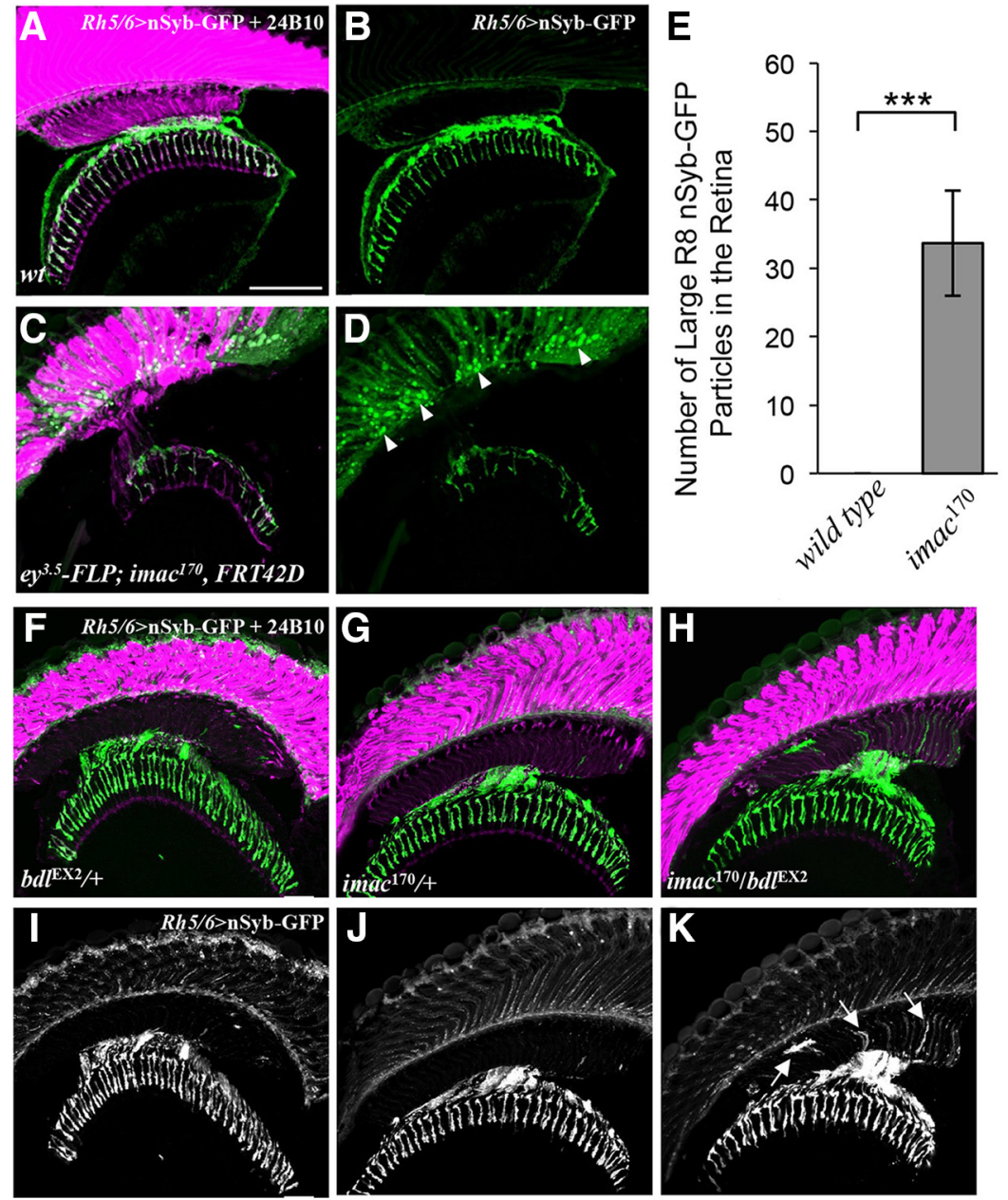

Figure 7. $\quad b d /$ interacts genetically with imac in the control of SV transport in R8 axons. A-D, Loss of imac caused a failure of SV components to transport from $R 8$ cell bodies into axons. Frozen sections of adult heads expressing UAS-nSybGFP under control of the green-sensitive-R8-specific driver Rh6-GAL4, were stained with anti-GFP (green) and MAb24B10 (magenta). $\boldsymbol{A}$, In wild-type ( $n=6$ animals), nSyb-GFP staining was predominantly localized to R8 axonal terminals in the medulla. $\boldsymbol{B}$, The section in $\boldsymbol{A}$ was visualized with nSyb-GFP staining only. $\boldsymbol{C}$, In eye-specific large imac ${ }^{170}$ homozygous clones ( $n=6$ animals), the levels of $n$ Syb-GFP staining in R8 axons were greatly reduced. A large number of nSyb-GFP-positive large aggregates were observed in R8 cell bodies in the retina. $\boldsymbol{D}$, The section in $\boldsymbol{C}$ was visualized with $n$ Syb-GFP staining only. $\boldsymbol{E}$, The number of abnormal large nSyb-GFP-positive


Student's $t$ test, ${ }^{* * *} p=0.0073$. Error Bars indicate SEM. $\boldsymbol{F}-\boldsymbol{K}$, Frozen sections of adult heads expressing UAS- $n$-Syb-GFP under control of the R8-specific driver Rh5/6-GAL4, were stained with anti-GFP (green) and MAb24B10 (magenta). In most bdl ${ }^{\mathrm{EX} 2} /+(\boldsymbol{F}$, I; 14 of 15 animals) or imac ${ }^{170} /+$ heterozygotes (G, J; 14 of 16 animals), nSyb-GFP staining was predominantly localized to R8 axonal terminals in the medulla region. In the majority of $b d^{\mathrm{EX} 2} /$ imac $^{170}$ transheterozygotes (12 of 15 animals), however, strong $\mathrm{n}$-Syb-GFP staining was also observed in the proximal portion of $\mathrm{R} 8$ axons in the lamina. Arrows indicate proximal portions of R8 axons with mislocalized nSyb-GFP. Scale bar, $20 \mu \mathrm{m}$.

in which Bdl-Bdl homophilic binding mediates specific interactions at axo-dendritic contact sites in promoting the activity and/or localization of Imac, and thus facilities axonal transport of presynaptic components in R8 axons (Fig. 8I).

Unlike several other well characterized cell adhesion molecules that control the development and function of the fly visual system (Clandinin et al., 2001; Lee et al., 2001, 2003; ShinzaKameda et al., 2006), Bdl is neither required for R-cell axon guidance nor layer-specific R-cell axonal target selection (Fig. 1; Cameron et al., 2013). In bdl-null mutants, both R7 and R8 axons projected through the lamina into their correct target layers in the medulla, where R7 and R8 axonal terminals are organized in regularly spaced columns (Cameron et al., 2013). By comparison, loss of either N-Cadherin or Lar causes R7 axons to project aberrantly into the R8 target layer (Clandinin et al., 2001; Lee et al.,
2001), whereas mutations in the gene $c a$ pricious encoding for a R8-specific cell adhesion molecule causes R8 targeting errors (Shinza-Kameda et al., 2006). Those studies indicate that Bdl is not involved in mediating the initial formation and/or maintenance of the contact between R8 axons and their targets in the medulla. Instead, Bdl may mediate specific interactions at axo-dendrite contact sites in the medulla, which is required for axonal transport of SV components in R8 axons.

How does Bdl function? In our previous studies (Cameron et al., 2013; Chen et al., 2017), we show that Bdl is capable of mediating both homophilic and heterophilic binding. Bdl on R8 axonal terminal may bind to Bdl on the target in a homophilic manner, thus mediates the interactions at axo-dendritic contact sites. Alternatively, an unknown cell-surface receptor on the target may bind to Bdl on the $\mathrm{R} 8$ presynaptic terminal in promoting axonal transport of SV components in R8 axons. Based on the results from transgene rescue and cell-type-specific knockdown experiments, we favor the first model in which Bdl mediates specific interactions at axo-dendritic contact sites via a homophilic binding mechanism (Fig. 8I).

That Bdl interacts genetically with Imac, together with that the levels of the Imac motor was significantly reduced in R8 presynaptic terminals, suggest strongly that Bdl functions directly or indirectly in regulating Imac. Bdl may regulate the function of Imac in several ways. First, Bdl may promote axonal localization of Imac. Unc-104/Imac/ KIF1A-family motor proteins exist in axons as either inactive state due to autoinhibition, or active state upon cargo binding (Hammond et al., 2009; Huo et al., 2012; Yue et al., 2013; Niwa et al., 2016). Whereas inactive KIF1A motors can diffuse on microtubules in the absence of cargo, active KIF1A motors undergo ATP-dependent processive motility in the presence of cargo (Hammond et al., 2009). Thus, the Bdl-dependent pathway may promote diffusion of inactive Imac on microtubules along R8 axons, which makes the motors available locally to respond to activating signals for delivering SV components to the presynaptic terminal (Fig. 8I). Second, the Bdl-dependent pathway may not only promote axonal localization of Imac, but also stimulate its activity. Consistent with this possibility, we found that overexpression of Imac in R8 axons was not sufficient for rescuing the SV phenotype in $b d l$ mutants (data not shown). A number of studies show that motor proteins can be activated by relieving the autoinhibition with cargo binding and phosphorylation (Espeut et al., 2008; Hammond et al., 2009; Niwa et al., 2016). Similarly, we speculate that Bdl may activate downstream signaling events, which may unlock the autoinhibition 

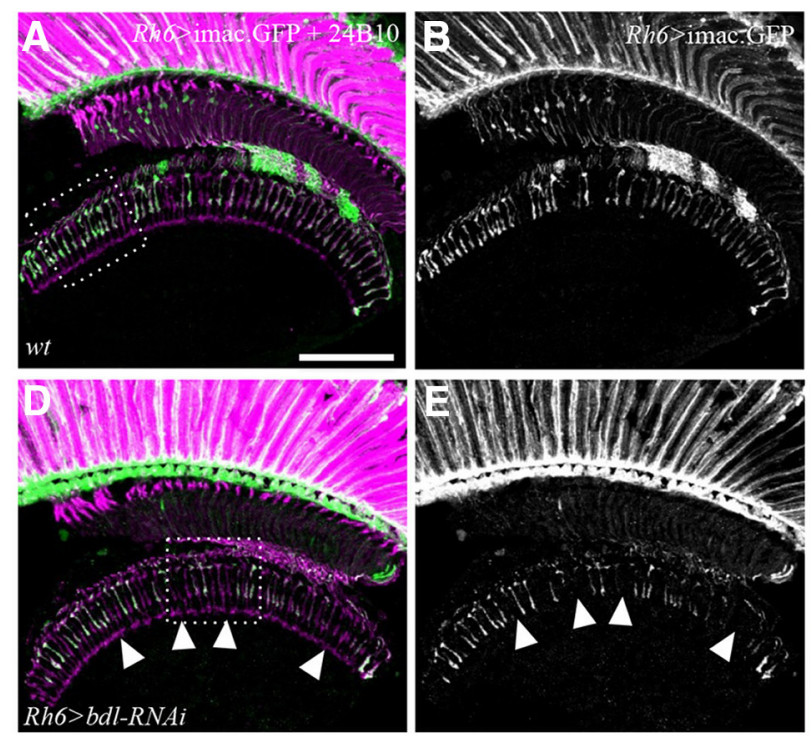

$$
\text { 管 }
$$

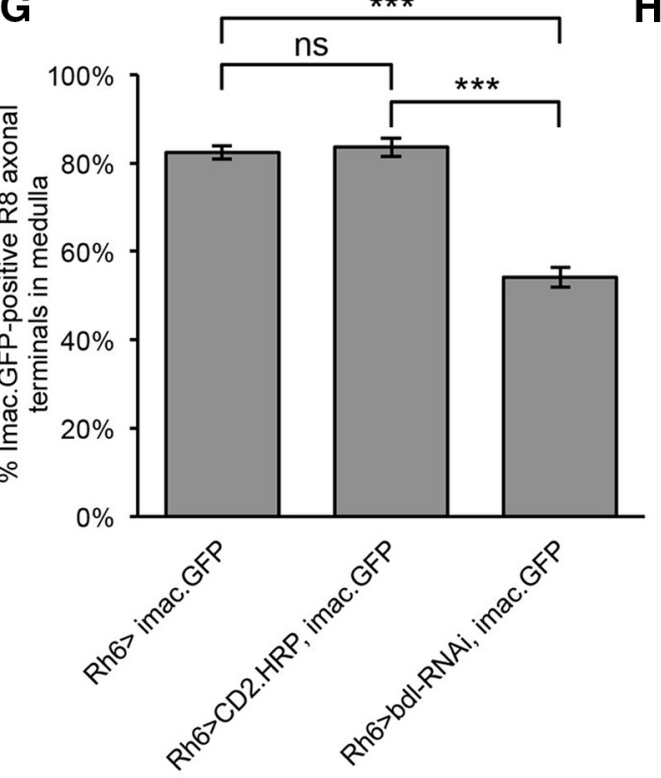

H

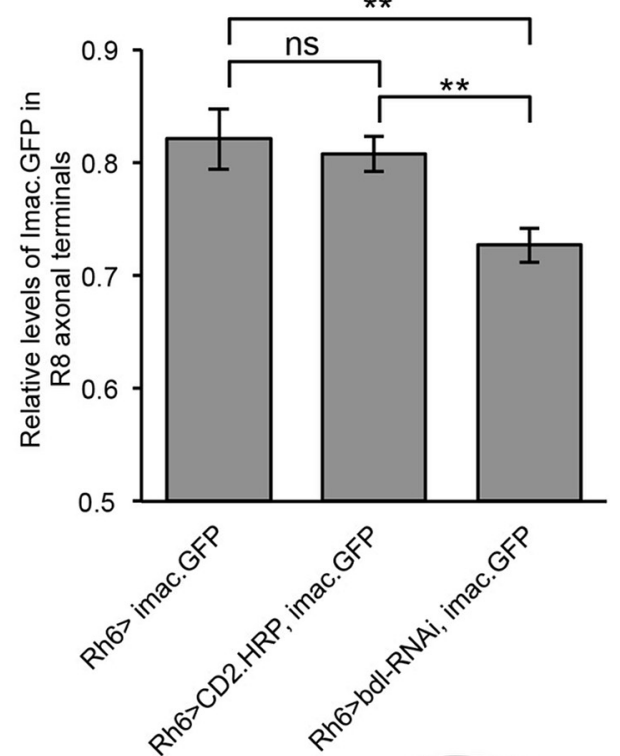

I

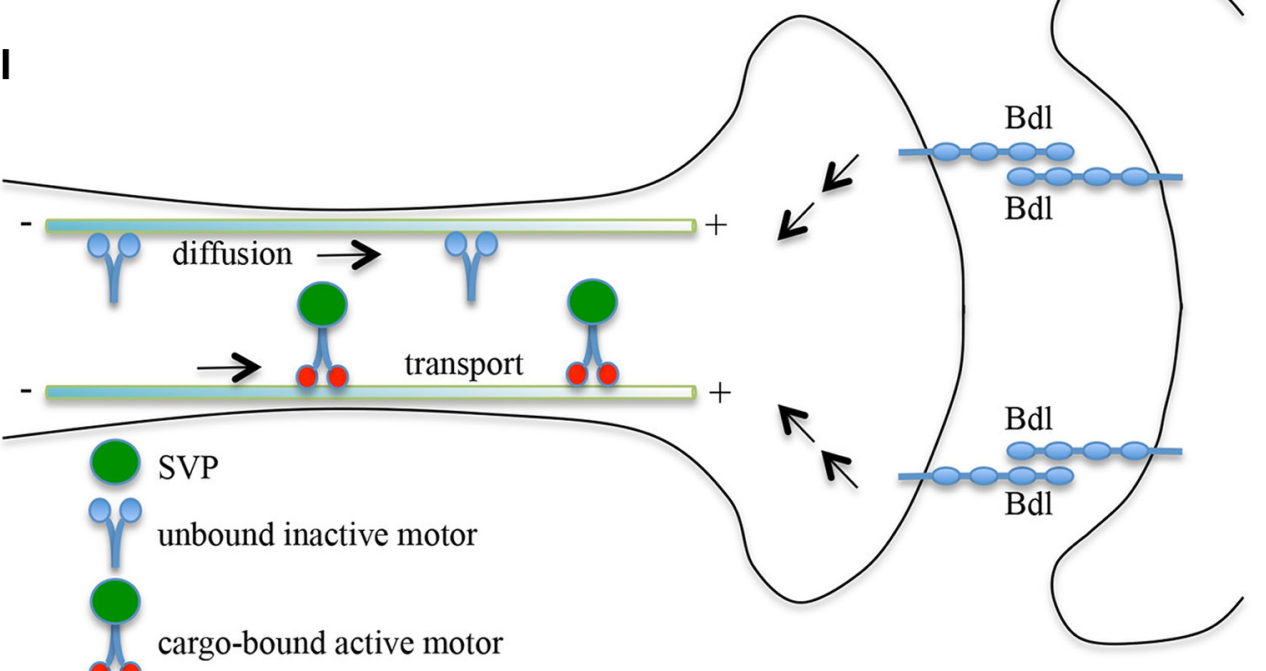

Figure 8. The levels of the Imac motor protein in R8 axonal terminals were decreased in bdl mutants. $\boldsymbol{A}-\boldsymbol{F}$, Frozen sections of adult heads expressing UAS-imac.GFP under control of the green-sensitive-R8-specific driver Rh6-GAL4, were stained with anti-GFP (green) and MAb24B10 (magenta). A, In control animals carrying Rh6-GAL4 and UAS-imac.GFP, Imac.GFP staining was observed in proximal portions of R8 axons in the lamina and R8 axonal terminals in the medulla. $\boldsymbol{B}$, The section in $\boldsymbol{A}$ was visualized with Imac.GFP staining only. $\boldsymbol{C}$, The boxed area in $\boldsymbol{A}$ was enlarged. $\boldsymbol{D}$, In $b d l$ knockdown flies carrying Rh6-GAL4, UAS-imac.GFP and UAS-bdl-RNAi, the number of R8 axonal terminals with Imac.GFP decreased. $\boldsymbol{E}$, The section in $\boldsymbol{D}$ was (Figure legend continues.) 
of Imac and thus promote the delivery of SV components to presynaptic terminals (Fig. 8I).

Our results also suggest a role for Bdl in regulating axonal transport of the AZ protein Brp. AZ components are transported in PTVs, which like SVPs, also rely on the kinesin-3 family motor Unc-104/Imac/KIF1A for axonal transport (Hall and Hedgecock, 1991; Otsuka et al., 1991; Okada et al., 1995; Zhao et al., 2001; Pack-Chung et al., 2007; Barkus et al., 2008; Niwa et al., 2008). Consistently, we show that loss of $b d l$ also affected the trafficking of Brp in R8 axons. In $b d l$ mutants, although many SV components were mislocalized to the proximal regions of R8 axons (Fig. 1), large Brp-positive aggregates accumulated abnormally in R8 soma close to the axonal initial segment (Fig. 4). That loss of $b d l$ affected the trafficking of SVs and Brp differently may be because of cargo difference. PTVs carrying AZ components have cargo size at $\sim 80 \mathrm{~nm}$ in diameter (Zhai et al., 2001; Tao-Cheng, 2007), whereas STVs are heterogeneous in size (Kraszewski et al., 1995; Ahmari et al., 2000). The difference in cargo size and/or surface properties of cargo may lead to different responses to altered motor function in $b d l$ mutants.

Although loss of $b d l$ affected the trafficking of SV and AZ components, axonal transport of mitochondria remained normal in $b d l$ mutants (Fig. 4). One likely explanation is that the transport of mitochondria in R8 axons uses a different motor system. It is reported that removal of the adapter protein Milton disrupts the transport of mitochondria, but not SVs, in Drosophila photoreceptor axons (Stowers et al., 2002). Milton directly interacts with the mitochondrial Rho-like GTPase Miro to recruit mitochondria to the kinesin-1 motor protein (Glater et al., 2006). Thus, it appears highly possible that unlike Imac-mediated transport of SVs, kinesin-1-mediated transport of mitochondria is regulated by a Bdl-independent signaling pathway.

$\mathrm{Bdl}$ is highly homologous to IgSF9A and IgSF9B in mammals (Hansen and Walmod, 2013). Interestingly, a recent in vitro study shows that IgSF9B forms a cis-complex with Neuroligin-2 (Nlg-2) on the postsynaptic membrane, and facilitates the transsynaptic interactions between Nlg-2 and Neurexin in mediating inhibitory synaptogenesis (Woo et al., 2013). IgSF9A knock-out study shows that loss of IgSF9A causes a reduction in the number of inhibitory synapses in the hippocampus (Mishra et al., 2014). In the future, it would be of interest to determine whether mammalian IgSF9 family proteins are also required for promoting axonal transport of SV components. Further molecular and genetic dissection of the Bdl-dependent pathway will provide novel and important insights into the general mechanisms underlying

\footnotetext{
$\leftarrow$

(Figurelegend continued.) visualized with Imac-GFP staining only. $\boldsymbol{F}$, The boxed area in $\boldsymbol{D}$ was enlarged. Arrowheads indicate R8 axonal terminals in which Imac.GFP was absent. G, The percentage of R8 axonal terminals with Imac.GFP staining was quantified. Compared with that in control animals carrying Rh6-GAL4 and UAS-imac.GFP ( $n=7$ animals) or control flies carrying Rh6-GAL4, UAS-imac.GFP and UAS-CD2.HRP ( $n=10$ animals), the percentage of R8 axonal terminals with Imac.GFP was significantly reduced in bdl knockdown flies carrying Rh6-GAL4, UAS-imac.GFP and UAS-bdl-RNAi ( $n=8$ animals). One-way ANOVA followed by post hoc Tukey's test, ${ }^{* * *} p<0.001 ; \mathrm{ns}, p>0.05$. $\boldsymbol{H}$, Relative intensity of Imac.GFP staining in Imac.GFP-positive R8 axonal terminals was quantified. Compared with that in control animals carrying Rh6-GAL4 and UAS-imac.GFP (101 Imac.GFP-positive R8 axons, $n=6$ animals) or control flies carrying Rh6-GAL4, UAS-imac.GFP and UAS-CD2.HRP (189 Imac.GFP-positive R8 axons, $n=6$ animals), relative intensity of Imac.GFP staining was reduced in $b d /$ knockdown $R 8$ axonal terminals (131 Imac.GFP-positive R8 axons, $n=7$ animals). One-way ANOVA followed by post hoc Tukey's test, ${ }^{* *} p<0.01 ; n s, p>0.05$. I, Proposal models for the action of Bdl. $\mathrm{Bdl}$-Bdl-mediated interaction between R8 and its postsynaptic target neuron may trigger downstream signaling events that promote the diffusion of inactive Imac and/or stimulates the activity of Imac. Scale bars: $\boldsymbol{A}, \boldsymbol{B}, \mathrm{D}, \boldsymbol{E}, 20 \mu \mathrm{m} ; \boldsymbol{C}, \boldsymbol{F}, 10 \mu \mathrm{m}$.
}

axonal transport of SV components for presynaptic assembly and function.

\section{References}

Ahmari SE, Buchanan J, Smith SJ (2000) Assembly of presynaptic active zones from cytoplasmic transport packets. Nat Neurosci 3:445-451.

Barkus RV, Klyachko O, Horiuchi D, Dickson BJ, Saxton WM (2008) Identification of an axonal kinesin-3 motor for fast anterograde vesicle transport that facilitates retrograde transport of neuropeptides. Mol Biol Cell 19:274-283.

Berger-Müller S, Sugie A, Takahashi F, Tavosanis G, Hakeda-Suzuki S, Suzuki $\mathrm{T}$ (2013) Assessing the role of cell-surface molecules in central synaptogenesis in the Drosophila visual system. PLoS One 8:e83732.

Bury LA, Sabo SL (2014) Dynamic mechanisms of neuroligin-dependent presynaptic terminal assembly in living cortical neurons. Neural Dev 9:13.

Bury LA, Sabo SL (2016) Building a terminal: mechanisms of presynaptic development in the CNS. Neuroscientist 22:372-391.

Byrd DT, Kawasaki M, Walcoff M, Hisamoto N, Matsumoto K, Jin Y (2001) UNC-16, a JNK-signaling scaffold protein, regulates vesicle transport in C. elegans. Neuron 32:787-800.

Cameron S, Chang WT, Chen Y, Zhou Y, Taran S, Rao Y (2013) Visual circuit assembly requires fine tuning of the novel Ig transmembrane protein borderless. J Neurosci 33:17413-17421.

Cameron S, Chen Y, Rao Y (2016) Borderless regulates glial extension and axon ensheathment. Dev Biol 414:170-180.

Chen Y, Cameron S, Chang WT, Rao Y (2017) Turtle interacts with borderless in regulating glial extension and axon ensheathment. Mol Brain 10: 17.

Chia PH, Li P, Shen K (2013) Cell biology in neuroscience: cellular and molecular mechanisms underlying presynapse formation. J Cell Biol 203: $11-22$.

Clandinin TR, Lee CH, Herman T, Lee RC, Yang AY, Ovasapyan S, Zipursky SL (2001) Drosophila LAR regulates R1-R6 and R7 target specificity in the visual system. Neuron 32:237-248.

Espeut J, Gaussen A, Bieling P, Morin V, Prieto S, Fesquet D, Surrey T, Abrieu A (2008) Phosphorylation relieves autoinhibition of the kinetochore motor cenp-E. Mol Cell 29:637-643.

Estes PS, Ho GL, Narayanan R, Ramaswami M (2000) Synaptic localization and restricted diffusion of a Drosophila neuronal synaptobrevin-green fluorescent protein chimera in vivo. J Neurogenet 13:233-255.

Gao S, Takemura SY, Ting CY, Huang S, Lu Z, Luan H, Rister J, Thum AS, Yang M, Hong ST, Wang JW, Odenwald WF, White BH, Meinertzhagen IA, Lee CH (2008) The neural substrate of spectral preference in Drosophila. Neuron 60:328-342.

Glater EE, Megeath LJ, Stowers RS, Schwarz TL (2006) Axonal transport of mitochondria requires milton to recruit kinesin heavy chain and is light chain independent. J Cell Biol 173:545-557.

Goldstein AY, Wang X, Schwarz TL (2008) Axonal transport and the delivery of pre-synaptic components. Curr Opin Neurobiol 18:495-503.

Hall DH, Hedgecock EM (1991) Kinesin-related gene unc-104 is required for axonal transport of synaptic vesicles in C. elegans. Cell 65:837-847.

Hammond JW, Cai D, Blasius TL, Li Z, Jiang Y, Jih GT, Meyhofer E, Verhey KJ (2009) Mammalian kinesin-3 motors are dimeric in vivo and move by processive motility upon release of autoinhibition. PLoS Biol 7:e72.

Hansen M, Walmod PS (2013) IGSF9 family proteins. Neurochem Res 38 : $1236-1251$.

Huo L, Yue Y, Ren J, Yu J, Liu J, Yu Y, Ye F, Xu T, Zhang M, Feng W (2012) The CC1-FHA tandem as a central hub for controlling the dimerization and activation of kinesin-3 KIF1A. Structure 20:1550-1561.

Klassen MP, Wu YE, Maeder CI, Nakae I, Cueva JG, Lehrman EK, Tada M, Gengyo-Ando K, Wang GJ, Goodman M, Mitani S, Kontani K, Katada T, Shen K (2010) An arf-like small G protein, ARL-8, promotes the axonal transport of presynaptic cargoes by suppressing vesicle aggregation. Neuron $66: 710-723$.

Kraszewski K, Mundigl O, Daniell L, Verderio C, Matteoli M, De Camilli P (1995) Synaptic vesicle dynamics in living cultured hippocampal neurons visualized with CY3-conjugated antibodies directed against the lumenal domain of synaptotagmin. J Neurosci 15:4328-4342.

Lee CH, Herman T, Clandinin TR, Lee R, Zipursky SL (2001) N-cadherin regulates target specificity in the Drosophila visual system. Neuron 30: 437-450.

Lee RC, Clandinin TR, Lee CH, Chen PL, Meinertzhagen IA, Zipursky SL 
(2003) The protocadherin flamingo is required for axon target selection in the Drosophila visual system. Nat Neurosci 6:557-563.

Macpherson LJ, Zaharieva EE, Kearney PJ, Alpert MH, Lin TY, Turan Z, Lee CH, Gallio M (2015) Dynamic labelling of neural connections in multiple colours by trans-synaptic fluorescence complementation. Nat Commun 6:10024.

Maeder CI, Shen K, Hoogenraad CC (2014) Axon and dendritic trafficking. Curr Opin Neurobiol 27:165-170.

McAllister AK (2007) Dynamic aspects of CNS synapse formation. Annu Rev Neurosci 30:425-450.

Mishra A, Traut MH, Becker L, Klopstock T, Stein V, Klein R (2014) Genetic evidence for the adhesion protein IgSF9/Dasm1 to regulate inhibitory synapse development independent of its intracellular domain. J Neurosci 34:4187-4199.

Nériec N, Desplan C (2016) From the eye to the brain: development of the Drosophila visual system. Curr Top Dev Biol 116:247-271.

Niwa S, Tanaka Y, Hirokawa N (2008) KIF1Bbeta- and KIF1A-mediated axonal transport of presynaptic regulator Rab3 occurs in a GTPdependent manner through DENN/MADD. Nat Cell Biol 10:1269-1279.

Niwa S, Lipton DM, Morikawa M, Zhao C, Hirokawa N, Lu H, Shen K (2016) Autoinhibition of a neuronal kinesin UNC-104/KIF1A regulates the size and density of synapses. Cell Rep 16:2129-2141.

Niwa S, Tao L, Lu SY, Liew GM, Feng W, Nachury MV, Shen K (2017) BORC regulates the axonal transport of synaptic vesicle precursors by activating ARL-8. Curr Biol 27:2569-2578.e4.

Okada Y, Yamazaki H, Sekine-Aizawa Y, Hirokawa N (1995) The neuronspecific kinesin superfamily protein KIF1A is a unique monomeric motor for anterograde axonal transport of synaptic vesicle precursors. Cell $81: 769-780$

Otsuka AJ, Jeyaprakash A, García-Añoveros J, Tang LZ, Fisk G, Hartshorne T, Franco R, Born T (1991) The C. elegans unc-104 gene encodes a putative kinesin heavy chain-like protein. Neuron 6:113-122.

Pack-Chung E, Kurshan PT, Dickman DK, Schwarz TL (2007) A Drosophila kinesin required for synaptic bouton formation and synaptic vesicle transport. Nat Neurosci 10:980-989.

Salcedo E, Huber A, Henrich S, Chadwell LV, Chou WH, Paulsen R, Britt SG (1999) Blue- and green-absorbing visual pigments of Drosophila: ectopic expression and physiological characterization of the R8 photoreceptor cell-specific Rh5 and Rh6 rhodopsins. J Neurosci 19:10716-10726.

Sanes JR, Zipursky SL (2010) Design principles of insect and vertebrate visual systems. Neuron 66:15-36.

Shinza-Kameda M, Takasu E, Sakurai K, Hayashi S, Nose A (2006) Regulation of layer-specific targeting by reciprocal expression of a cell adhesion molecule, capricious. Neuron 49:205-213.

Stowers RS, Megeath LJ, Górska-Andrzejak J, Meinertzhagen IA, Schwarz TL (2002) Axonal transport of mitochondria to synapses depends on milton, a novel Drosophila protein. Neuron 36:1063-1077.

Sugie A, Hakeda-Suzuki S, Suzuki E, Silies M, Shimozono M, Möhl C, Suzuki T, Tavosanis G (2015) Molecular remodeling of the presynaptic active zone of Drosophila photoreceptors via activity-dependent feedback. Neuron 86:711-725.

Tahayato A, Sonneville R, Pichaud F, Wernet MF, Papatsenko D, Beaufils P, Cook T, Desplan C (2003) Otd/Crx, a dual regulator for the specification of ommatidia subtypes in the Drosophila retina. Dev Cell 5:391-402.

Tao-Cheng JH (2007) Ultrastructural localization of active zone and synaptic vesicle proteins in a preassembled multi-vesicle transport aggregate. Neuroscience 150:575-584.

Ting CY, McQueen PG, Pandya N, Lin TY, Yang M, Reddy OV, O'Connor MB, McAuliffe M, Lee CH (2014) Photoreceptor-derived activin promotes dendritic termination and restricts the receptive fields of first-order interneurons in Drosophila. Neuron 81:830-846.

Van Vactor D Jr, Krantz DE, Reinke R, Zipursky SL (1988) Analysis of mutants in chaoptin, a photoreceptor cell-specific glycoprotein in Drosophila, reveals its role in cellular morphogenesis. Cell 52:281-290.

Vasiliauskas D, Mazzoni EO, Sprecher SG, Brodetskiy K, Johnston RJ Jr, Lidder P, Vogt N, Celik A, Desplan C (2011) Feedback from rhodopsin controls rhodopsin exclusion in Drosophila photoreceptors. Nature 479:108-112.

Wagh DA, Rasse TM, Asan E, Hofbauer A, Schwenkert I, Dürrbeck H, Buchner S, Dabauvalle MC, Schmidt M, Qin G, Wichmann C, Kittel R, Sigrist SJ, Buchner E (2006) Bruchpilot, a protein with homology to ELKS/ CAST, is required for structural integrity and function of synaptic active zones in Drosophila. Neuron 49:833-844.

Woo J, Kwon SK, Nam J, Choi S, Takahashi H, Krueger D, Park J, Lee Y, Bae JY, Lee D, Ko J, Kim H, Kim MH, Bae YC, Chang S, Craig AM, Kim E (2013) The adhesion protein IgSF9b is coupled to neuroligin 2 via S-SCAM to promote inhibitory synapse development. J Cell Biol 201:929-944.

Wu YE, Huo L, Maeder CI, Feng W, Shen K (2013) The balance between capture and dissociation of presynaptic proteins controls the spatial distribution of synapses. Neuron 78:994-1011.

Yamaguchi S, Desplan C, Heisenberg M (2010) Contribution of photoreceptor subtypes to spectral wavelength preference in Drosophila. Proc Natl Acad Sci U S A 107:5634-5639.

Yue Y, Sheng Y, Zhang HN, Yu Y, Huo L, Feng W, Xu T (2013) The CC1FHA dimer is essential for KIF1A-mediated axonal transport of synaptic vesicles in C. elegans. Biochem Biophys Res Commun 435:441-446.

Zhai RG, Vardinon-Friedman H, Cases-Langhoff C, Becker B, Gundelfinger ED, Ziv NE, Garner CC (2001) Assembling the presynaptic active zone: a characterization of an active one precursor vesicle. Neuron 29:131-143.

Zhang YQ, Rodesch CK, Broadie K (2002) Living synaptic vesicle marker: synaptotagmin-GFP. Genesis 34:142-145.

Zhao C, Takita J, Tanaka Y, Setou M, Nakagawa T, Takeda S, Yang HW, Terada S, Nakata T, Takei Y, Saito M, Tsuji S, Hayashi Y, Hirokawa N (2001) Charcot-marie-tooth disease type $2 \mathrm{~A}$ caused by mutation in a microtubule motor KIF1Bbeta. Cell 105:587-597.

Zong W, Wang Y, Tang Q, Zhang H, Yu F (2018) Prd1 associates with the clathrin adaptor $\alpha$-Adaptin and the kinesin-3 Imac/Unc-104 to govern dendrite pruning in Drosophila. PLoS Biol 16:e2004506. 